\title{
G

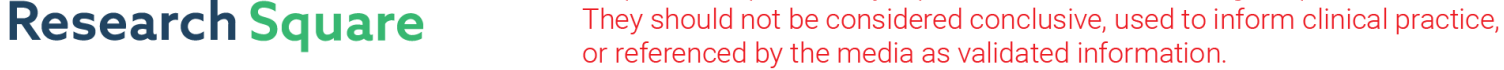 \\ Link ER ion homeostasis maintained by an ER anion channel to ALS
}

\section{Yichang Jia ( $\nabla$ yichangjia@mail.tsinghua.edu.cn )}

Tsinghua University

\section{Liang Guo}

Tsinghua-Peking Joint Center for Life Sciences, Tsinghua University, Beijing, 100084, China.

\section{Qionglei Mao}

Shanghai Institute of Materia Medica, Chinese Academy of Sciences.

Ji He

Peking University Third Hospital

\section{Xiaoling LIU}

School of Pharmaceutical Sciences, Tsinghua University

\section{Xuejiao Piao}

Tsinghua University

\section{Li Luo}

Tsinghua University

\section{Xiaoxu Hao}

Zhejiang University

\section{Bailong Xiao}

State Key Laboratory of Membrane Biology, Tsinghua-Peking Joint Center for Life Sciences, Beijing Advanced Innovation Center for Structural Biology, IDG/McGovern Institute for Brain Research, School https://orcid.org/0000-0002-2386-3322

\section{Dongsheng Fan}

Department of Neurology, Peking University Third hospital

\section{Zhaobing Gao}

Shanghai Institute of Materia Medica, Chinese Academy of Sciences

\section{Biological Sciences - Article}

Keywords: Anion channel, endoplasmic reticulum (ER), ion homeostasis, ER stress, ALS.

Posted Date: May 27th, 2021

DOI: https://doi.org/10.21203/rs.3.rs-536643/v1 
License: (c) (i) This work is licensed under a Creative Commons Attribution 4.0 International License. Read Full License 
Title: Link ER ion homeostasis maintained by an ER anion channel to ALS

\section{Authors/Affiliations :}

Liang Guo ${ }^{1,3,4,5 *}$, Qionglei Mao ${ }^{2,6 *}$, Ji He ${ }^{7 *}$, Xiaoling $\mathrm{Liu}^{5,8}$, Xuejiao Piao ${ }^{1,3,4,5}$, Li

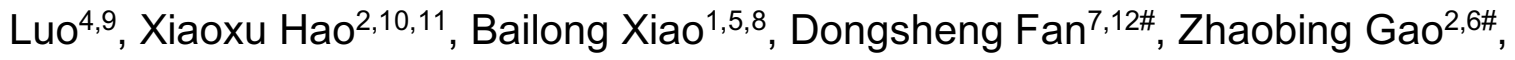
and Yichang Jia ${ }^{1,4,5,9 \#}$

${ }^{1}$ Tsinghua-Peking Joint Center for Life Sciences, Tsinghua University, Beijing, 100084, China. ${ }^{2}$ CAS Key Laboratory of Receptor Research, State Key Laboratory of Drug Research, Shanghai Institute of Materia and Medica, Chinese Academy of Sciences, 555 Zuchongzhi Road, Shanghai, 201203, China.

${ }^{3}$ School of Life Sciences, Tsinghua University, Beijing, 100084, China.

${ }^{4}$ School of Medicine, Tsinghua University, Beijing, 100084, China.

${ }^{5}$ IDG/McGovern Institute for Brain Research, Tsinghua University, Beijing, 100084, China.

${ }^{6}$ University of Chinese Academy of Sciences, No.19 (A) Yuquan Road, Beijing, 100049, China ${ }^{7}$ Department of Neurology, Peking University Third Hospital, Beijing, 100191, China.

${ }^{8}$ School of Pharmaceutical Sciences, Tsinghua University, Beijing, 100084, China.

${ }^{9}$ Tsinghua Laboratory of Brain and Intelligence, Beijing, 100084, China

${ }^{10}$ School of Pharmaceutical Sciences, Zhejiang University, Hangzhou, 310058, China.

${ }^{11}$ School of Medicine, Zhejiang University City College, Hangzhou, 310015, China.

${ }^{12}$ Beijing Municipal Key Laboratory of Biomarker and Translational Research in Neurodegenerative Diseases, Beijing, 100191, China

*These authors contributed equally to this work.

\# Corresponding authors

Please address correspondence to: Dongsheng Fan, MD, PhD

Neurological Department of Peking University Third Hospital, Haidian District, Beijing No. 49, North Garden Road, Haidian District, Beijing, 100191, China.

Tel: 86-10-82265032, Email: dsfan2010@aliyun.com

\# Corresponding authors

Please address correspondence to: Zhaobing Gao, Ph. D.

CAS Key Laboratory of Receptor Research, State Key Laboratory of Drug Research, Shanghai Institute of Materia Medica, Chinese Academy of Sciences, 555 Zuchongzhi Road, Shanghai, 201203, China.

Tel: 86-21-20239067, Email: zbgao@simm.ac.cn

\# Corresponding author

Please address correspondence to: Yichang Jia, Ph. D.

School of Medicine, Medical Science Building, Room D204, Tsinghua University, Beijing, 100084, P. R. China

Tel: 86-10-62781045, Email: yichangjia@tsinghua.edu.cn 


\section{Abstract: (200 words)}

Although anion channel activities have been demonstrated in sarcoplasmic reticulum/endoplasmic reticulum (SR/ER), their molecular identities and functions remain unclear. Here, we link rare variants of CLCC1 (Chloride Channel CLIC Like 1) to ALS (amyotrophic lateral sclerosis). We demonstrate that CLCC1 is a pore-forming component of an ER anion channel and that ALS-associated mutations impair the channel activity. CLCC1 unitary conductance is inhibited by luminal $\mathrm{Ca}^{2+}$ but facilitated by phosphatidylinositol 4,5-bisphosphate (PIP2). We identified a conserved lysine 298 (K298) in CLCC1 intraluminal loop as the critical PIP2-sensing residue. CLCC1 maintains steady-state $\left[\mathrm{Cl}^{-}\right]_{E R}$ and morphology and regulates $\mathrm{ER} \mathrm{Ca}{ }^{2+}$ homeostasis including steady-state $\left[\mathrm{Ca}^{2+}\right]_{E R}$ and efficiency of internal $\mathrm{Ca}^{2+}$ release. ALS-associated mutant CLCC1 increase steady-state $\left[\mathrm{Cl}^{-}\right]_{E R}$ and impair ER $\mathrm{Ca}^{2+}$ homeostasis. Phenotypic comparisons of multiple Clcc1 mutant alleles, including ALS-associated mutations, reveal a CLCC1 dosage-dependence in severity of disease phenotypes in vivo. Conditional knockout of Clcc1 cell-autonomously causes motor neuron loss and ER stress, misfolded protein accumulation, and characteristic ALS pathologies in the spinal cord. Thus, we argue that disruption of ER ion homeostasis maintained by CLCC1 underlies etiology of neurodegenerative diseases.

\section{Keywords:}

Anion channel, endoplasmic reticulum (ER), ion homeostasis, ER stress, ALS. 
Although $\mathrm{Cl}^{-}$is the most abundant anion in living cells, chloride currents and their functional significance had been understudied until the CLC family of chloride channels and CFTR (cystic fibrosis transmembrane conductance regulator) were cloned and their dysfunctions were linked to human diseases ${ }^{1-4}$. In addition to those on the cell surface, $\mathrm{Cl}^{-}$channels have long been proposed to exist in the intracellular membrane-bound organelles ${ }^{3,5}$. However, the previously postulated intracellular $\mathrm{Cl}^{-}$channels, like CLCAs (chloride channel $\mathrm{Ca}^{2+}$-activated) and CLICs (chloride intracellular channels), are now considered not likely to function as anion channels ${ }^{6,7}$. Therefore, the molecular identities and functions of organellar anion channels, including those in the SR/ER, remain largely unknown.

As the major internal $\mathrm{Ca}^{2+}$ store, $\mathrm{Ca}^{2+}$ release from $\mathrm{SR} / \mathrm{ER}$ is mediated mainly by two cation channels, RyRs (ryanodine receptors) and IP3Rs (inositol 1,4,5trisphosphate receptors) ${ }^{8-10}$. Other cation channels in SR/ER membranes regulate the $\mathrm{Ca}^{2+}$ release/content through different mechanisms ${ }^{11}$. For example, TRICs (TRimeric Intracellular Cation channels) are potassium channels that regulate $\mathrm{Ca}^{2+}$ release via a counter-ion mechanism, in which the influx of $\mathrm{K}^{+}$ through TRICs balances the loss of positive charges from the SR/ER as a result of the $\mathrm{Ca}^{2+}$ efflux, which helps maintain the driving force for continued $\mathrm{Ca}^{2+}$ release 12. In addition to cations, anions have also been proposed to regulate $\mathrm{ER} \mathrm{Ca}^{2+}$ release through the counter-ion mechanism, and various $\mathrm{Cl}^{-}$channel activities 
83 have been long demonstrated in microsome preparations ${ }^{11,13-17}$. A previous

84 study using mouse forward genetics revealed that loss of CLCC1 (Chloride

85 Channel CLIC Like 1), an ER resident protein, leads to ER stress and

86 neurodegeneration ${ }^{18}$. However, despite the name, CLCC1 has little sequence

87 similarity with CLIC family members or any known ion channels. In addition,

88 question remains whether the recorded chloride currents in microsome prepared

89 from the CLCC1 overexpressing cells were actually mediated by CLCC1 19.

90 Therefore, further evidence is needed to know if CLCC1 functions as an anion

91 channel.

92 ER stress and its related misfolded protein accumulation are one of the central

93 pathogenic pathways underlying neurodegenerative diseases, including ALS ${ }^{20-22 .}$

94 Here, we link CLCC1 rare mutations to ALS and demonstrate CLCC1 is the pore-

95 forming component of an ER anion channel. Dysfunction of CLCC1 impairs

96 steady state $\mathrm{ER}\left[\mathrm{Cl}^{-}\right]$and misregulates $\mathrm{ER} \mathrm{Ca}^{2+}$ homeostasis and leads to ER

97 swelling, ER stress, and protein misfolding. Therefore, we argue that

98 misregulation of ER ion homeostasis maintained by CLCC1 underlies etiology of

99 neurodegenerative diseases. 
100 Rare genetic variances in CLCC1 found in a Chinese ALS cohort

101 To identify novel rare mutations potentially associated with ALS, we performed

102 whole exome sequencing in a Chinese cohort (670 sporadic ALS patients and

1031910 controls). We identified 9 rare variances in CLCC1 in the patients, including

1047 nonsynonymous and 2 stopgain mutations (Fig. 1a, Extended Data Fig. 1, and

105 Supplementary Table 1). Among the mutations (Fig. 1b), the S263R and W267R

106 mutations have not been found in the public databases nor in our controls

107 (Supplementary Table 1). No mutations in known ALS-causing genes were

108 detected in the patients carrying S263R or W267R mutation. Notably, two

109 geographically and genetically unrelated patients with similar clinical phenotypes

110 shared the same S263R mutation (Supplementary Table 1). The two mutations

111 change Ser and Try to Arg, suggesting that they perturb local steric hindrance and

112 surface potential. CLCC1 is ubiquitously expressed and its disruption has been

113 shown to lead to ER stress and neurodegeneration in mice ${ }^{18}$. A burden analysis

$114{ }^{23}$ was further carried out and revealed that CLCC1 is associated with ALS $(p=$

$1151.51 \times 10^{-6}$, with OR $=5.72$ ), reaching suggestive significance (Fig. 1c).

116 ALS mutations S263R and W267R reduce CLCC1 expression and promote

117 ER stress and protein misfolding in vivo

118 Homozygous knockout of Clcc1 in mice is lethal, indicating Clcc1 is essential

119 (Extended Data Fig. 2a and Supplementary Table 2). Evolutionarily, CLCC1 
120 orthologues appear in vertebrate bot not invertebrate (Fig. 1d). Human and

121 mouse CLCC1 share 73\% identity, but S263 and W267 are conserved between

122 the two species. To examine the biological consequence of S263R and W267R

123 in vivo, we generated S263R and W267R knock-in mouse lines (Extended Data

124 Fig. 2b and 2c). Mice heterozygous for S263R and W267R were viable and fertile,

125 and no obvious ER stress and protein misfolding was disclosed in S263R

126 heterozygous mutant (S263R/+) cerebella (Fig. 1e). However, Bip upregulation

127 and ubiquitin-positive misfolded protein accumulation were documented in the

128 cerebella of mice compound heterozygous for S263R and the NM2453 allele

$129(\mathrm{~S} 263 \mathrm{R} / \mathrm{NM})$ - where an IAP (intracisternal A-particle) insertion in the intron 2 of

130 Clcc1 greatly reduces the expression of CLCC1 protein to $\sim 10 \%$ of that in wildtype

131 animals ${ }^{18}$. Like S263R/NM mutants, the W267R/NM mutants displayed the

132 similar extent of ER stress and protein misfolding in cerebella (Fig. 1e). In

133 contrast, mice heterozygous for $N M 2453$ allele $(N M /+)$ were normal without ER

134 stress in cerebella ${ }^{18}$. In addition to the pathologies, both S263R and W267R

135 mutations reduced mutant CLCC1 expression to a similar extent, suggesting that

136 severity of mutant Clcc1 phenotypes depends on the dosage of CLCC1 protein

137 (Fig. 1f). Indeed, we failed to harvest W267R/KO pup, suggesting that the ALS-

138 associated mutant alleles are functionally damaging in vivo, which is independent

139 of NM2453 allele (Supplementary Table 2). Therefore, our data support the 
140 notion that ALS-associated S263R and W267R mutations are potential disease-

141 causing.

\section{CLCC1 forms homomultimer in the ER membrane}

143 Based on its primary sequence, CLCC1 shares little sequence similarity with any

144 known ion channel and is predicted to contain three transmembrane segments

145 (TMs) and an N-terminal signal peptide (Fig. 2a). We generated antibodies

146 against the $\mathrm{N}$ - and C-termini of CLCC1 (Extended Data Fig. 3a). Using the C-

147 terminal antibody, we confirm that as suggested by a previous report ${ }^{18}$ CLCC1 is

148 predominantly ER-localized, as demonstrated by its co-localization with

149 CALNEXIN, an ER resident protein (Extended Data Fig. 3b).

150 To understand how CLCC1 functions in the ER, we treated human 293FT cells

151 with disuccinimidyl suberate (DSS), a crosslinker with a spacer length of $11.4 \AA$.

152 The C-terminal antibody detected high molecular weight complexes in a DSS

153 dosage-dependent manner from whole cell lysate. From the complex sizes, we

154 speculated that CLCC1 forms homomultimers (Fig. 2b), which was supported by

155 co-immunoprecipitations of differentially tagged CLCC1 co-expressed in the same

156 cells (Extended Data Fig. 4a) and of exogenous tagged CLCC1 with endogenous

157 CLCC1 (Extended Data Fig. 4b). In addition, purified CLCC1 N- and C-terminal

158 polypeptides formed homomultimers in vitro in a DSS-dependent manner

159 (Extended Data Fig. 4c and 4d), and disulfide bonds between cysteine residues 
160

161

162

163

164

165

166

167

168

169

170

171 Incorporation of the purified full-length mCLCC1 (Fig. 2c) into planar lipid bilayer

172 resulted in frequent inward currents at $0 \mathrm{mV}(-2.2 \pm 0.1 \mathrm{pA})$ in asymmetric $\mathrm{KCl}$

173 solutions (In/Ex, 150/15 mM) and the currents became outward at $90 \mathrm{mV}(1.6 \pm$

$1740.1 \mathrm{pA})$ (Fig. 2d). As a negative control, the protein purification buffer without

175 protein gave rise to no current (Fig. 2d). Based on the fit of the current-voltage

176 relationship, the reversal potentials were determined to be $56.8 \mathrm{mV}$ (In/Ex, 150/15

$177 \mathrm{mM} \mathrm{KCl})$ and $-60.3 \mathrm{mV}(\mathrm{In} / \mathrm{Ex}, 15 / 150 \mathrm{mM} \mathrm{KCl})$, which are close to the calculated

178 values for $\mathrm{Cl}^{-}$by Nernst equation, and the slope conductance was $39.9 \pm 1.0 \mathrm{pS}$

179 (mean \pm SEM). The permeability ratio of $P\left(\mathrm{Cl}^{-}\right)$to $P\left(\mathrm{~K}^{+}\right)$is about 100 to 1 and 
180 similar results were obtained by using asymmetric $\mathrm{NaCl}$ solutions (Fig. 2d).

181 Consistent with the single channel results, the reversal potential obtained from 182 studying macroscopic currents was $61.9 \mathrm{mV}$ in 15/150 mM KCl (In/Ex) (Extended 183 Data Fig. 5), further supporting the anion selectivity.

184 Next, we examined CLCC1 channel permeability to various anions, including $185 \mathrm{Br}^{-}, \mathrm{SO}_{4}{ }^{2-}$, and $\mathrm{NO}_{3}^{-}$, by adding $150 \mathrm{mM} \mathrm{KCl}$ in cis (In) and equal electric charges 186 of $\mathrm{KBr}, \mathrm{K}_{2} \mathrm{SO}_{4}$, or $\mathrm{KNO}_{3}$ in the trans (Ex) chamber (Fig. 2e). The relative 187 permeabilities of these anions to $\mathrm{Cl}^{-}$were $16.51\left(P_{\mathrm{Br}} / P_{\mathrm{Cl}}\right), 1.55\left(P_{\mathrm{SO}} / P_{\mathrm{Cl}}\right)$, and 0.22 $188\left(P_{\mathrm{NO} 3} / P_{\mathrm{Cl}}\right)$, respectively, indicating a sequence of the CLCC1 anion selectivity of $189 P_{\mathrm{Br}}>P_{\mathrm{SO} 4}>P_{\mathrm{Cl}}>P_{\mathrm{NO} 3 .}$ In these experiments, no cation permeation was detected.

190 To examine how S263R and W267R alter CLCC1 channel activity, we 191 incorporated the purified human wildtype (hWT), S263R or W267R mutant CLCC1 192 proteins into the lipid bilayer for single channel analysis. The slope conductances 193 of both S263R and W267R were significantly lower than that of hWT (Fig. 2f). 194 Collectively, our results demonstrate that CLCC1 is a pore-forming component of 195 an anion channel and that ALS-associated mutations impair CLCC1 unitary 196 conductance.

\section{ER membrane topology of CLCC1 and its inhibition by luminal calcium}

198 To examine CLCC1 topology in the ER membrane, we treated microsomes 199 prepared from wildtype mouse cerebella and livers ${ }^{25}$ with trypsin and analyzed the 
remaining CLCC1 fragments with our $\mathrm{N}$ - and $\mathrm{C}$-terminal antibodies (Extended Data

Fig. 3a). In the absence of Triton X-100, the N-terminus and the first and second loops of CLCC1 and an ER lumen resident protein Bip were protected from trypsinization, but the C-terminus of CLCC1 was not (Fig. $2 g$ and Extended Data Fig. 3c). As expected for membrane enclosure, the protection was disrupted by Triton X-100, suggesting that CLCC1 N-terminus and the second loop reside in ER lumen while C-terminus faces cytoplasm.

Interestingly, when we applied MTSET (methanethiosulfonateethyltrimethylammonium) ${ }^{26}$, a membrane-impermeant thiol reagent that modifies cysteine residues, in trans but not to the cis side of the chamber we applied the

210 purified CLCC1, the CLCC1 currents were suppressed (Fig. 2h), suggesting that a 211 specific orientation of CLCC1 in the bilayer is responsible for the current. Based 212 on the topology (Fig. $2 \mathrm{~g}$ ), cysteine residues are located in both the cytoplasm and 213 ER lumen sides of CLCC1 and C350 lies at the end of TM3 (Fig. 2i). Protein 214 alignment among different species revealed that C350 is in a consecutive row of 215 four residues (F $\underline{C} Y G)$, although it is less conserved than the other three

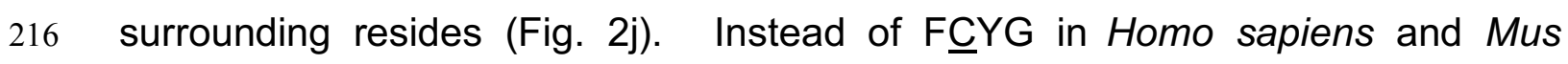
217 musculus, FEYG appears in Xenopus tropicalis, which prompted us to mutate

218 C350 to F. C350F mCLCC1 is expressed and its chromatographic behavior is 219 similar to wildtype mCLCC1 (Extended Data Fig. 6a). Importantly, C350F 
restored the CLCC1 currents even when MTSET was applied in trans side (Fig. 2h), suggesting that MTSET acts on C350 to modify the channel activity and the trans side is the CLCC1 cytoplasm side in the reconstructed lipid bilayer. Application of DIDS (4,4'-Diisothiocyano-2,2'-stilbenedisulfonic acid), a chloride transporter/channel blocker ${ }^{4,27}$, significantly inhibited CLCC1 channel activity (Extended Data Fig. 6b and 6c). Consistent with MTSET acting on C350, C350F largely restores the DIDS inhibition on channel open probability $\left(P_{\mathrm{o}}\right)$, suggesting that $\mathrm{C} 350$ is close to the CLCC1 conduction pathway.

Because ER luminal $\mathrm{Ca}^{2+}$ is much higher than cytoplasm, we then asked whether $\mathrm{Ca}^{2+}$ is able to differentially regulate $\mathrm{CLCC} 1$ channel activity from ER luminal or cytoplasmic side. Application of $\mathrm{Ca}^{2+}$ in cis/ER lumen side blocked the CLCC1 channel activity, which could be partially rescued by addition of equal molar EGTA, a $\mathrm{Ca}^{2+}$ chelating agent (Fig. 2k and 2l). However, the same application in trans/cytoplasm side had no effect on the channel activity. Therefore, we conclude that, at least in our reconstructed lipid bilayer setting, high concentration of $\mathrm{Ca}^{2+}$ at the ER lumen side inhibits CLCC1 channel activity.

\section{CLCC1 maintains steady state $\left[\mathrm{Cl}^{-}\right]_{\mathrm{ER}}$ and ER morphology}

To examine whether $\mathrm{CLCC} 1$ is involved in regulation of $[\mathrm{Cl}]_{E R}$, we employed a previously optimized YFP Cl${ }^{-}$sensor that responds to $\mathrm{Cl}^{-}$concentration change with super sensitivity and photostability ${ }^{28}$. To create a ratiometric $\mathrm{ER} \mathrm{Cl}^{-}$sensor, we built a signal sequence, a DsRed internal control, and an ER retention motif 
241 into the $\mathrm{Cl}^{-}$sensor, which we named RaMoride ER (Fig. 3a). ER localization of

242 RaMoride ${ }^{\mathrm{ER}}$ was confirmed by its colocalization with ER resident protein

243 CALNEXIN (Fig. 3b). The ratio between YFP to Ds-Red signals responded 244 correspondingly when extracellular $\left[\mathrm{Cl}^{-}\right]\left(\left[\mathrm{Cl}^{-}\right]\right.$Extra $)$was switched from $140 \mathrm{mM}$ to 245100 or 0 mM (Extended Data Fig. 7a and 7b).

246 To examine ALS-associated rare variants on [Cl- $]_{\mathrm{ER}}$, we expressed $\mathrm{hWT}$, M29T, 247 S263R, or W267R mutant CLCC1 in 293FT cells stably expressing RaMoride ${ }^{\mathrm{ER}}$ 248 together with an engineered near-infrared fluorescent protein, miRFP670S ${ }^{29}$, 249 which allowed us to sort the CLCC1 expressing cells but not disturbing the 250 RaMoride ${ }^{E R}$ signals (Fig. 3a and 3c). Compared to hWT, S263R or W267R but 251 not M29T mutant CLCC1 significantly increased steady state $\left[\mathrm{Cl}^{-}\right]_{E R}$, supporting 252 that S263R and W267R are functionally damaging mutations (Fig. 3c and 3d). 253 Consistent with the essential role of CLCC1 in vivo, we failed to generate a CLCC1 254 KO 293FT cell line by Crispr/Cas9. Instead, we knocked down CLCC1 with two 255 individual shRNAs ( $\mathrm{H} 3$ and $\mathrm{H} 4)$ (Fig. 3e). Although the two shRNAs had different 256 CLCC1 knockdown efficiencies (for $\mathrm{H} 3,22.5 \pm 0.6 \%$ of scrambled control; for $\mathrm{H} 4$, $25745.25 \pm 2.1 \%$ of scrambled control), both of them significantly increased steady 258 state $\left[\mathrm{Cl}^{-}\right]_{E R}$ to a similar extent in comparison with scrambled shRNA control (Fig. 259 3f).

260 The concentration of electrically charged osmolytes, such as $\mathrm{Cl}^{-}$, inside a cell 261 or intracellular membrane-bound organelle governs the volume of the 
262 compartment ${ }^{5,30}$. Therefore, we asked whether depletion of CLCC1 changes ER

263 volume. To this end, we collected 293FT cells expressing scrambled control or

264 CLCC1 shRNAs and applied for transmission electron microscopy (TEM) (Fig. $3 g$

265 and 3h). Enlarged and stubby ER morphology was documented in cells 266 expressing the individual CLCC1 shRNA. In contrast, ribosome-bound and

267 tubule-like ER was shown in the scrambled controls. In order to quantitatively 268 reflect ER morphology, we measured ER width in these three groups of cells. ER 269 width in the two individual CLCC1 shRNA groups was significantly increased as 270 compared to scrambled shRNA control. ER width in H3 shRNA group, where

271 there is higher knockdown efficiency, was significantly larger than that in $\mathrm{H} 4$ 272 shRNA group, suggesting a CLCC1 dosage-dependence in the effect on ER 273 swelling.

274 CLCC1 facilitates internal $\mathrm{Ca}^{2+}$ release and ALS-associated mutations impair 275 the $\mathrm{Ca}^{2+}$ release

276 ER-localized ion channels have been proposed to control ER $\mathrm{Ca}^{2+}$ mobilization 277 through a counter-ion mechanism 11,14,16,17. We then asked whether as an ER 278 chloride channel CLCC1 is involved in regulation of ER Ca ${ }^{2+}$ release. Knockdown 279 of CLCC1 by two individual shRNAs markedly reduced internal $\mathrm{Ca}^{2+}$ release 280 induced by ATP (Fig. 4a), which triggers ER Ca ${ }^{2+}$ release by generating IP3 that 281 activates IP3Rs ${ }^{10}$. Compared to mock control and scrambled shRNA, 
282 knockdown of CLCC1 by two individual shRNA not only significantly reduced the 283 amplitude, but also the rate (as reflected by the increase in time-to-peak), of ATP284 induced $\mathrm{Ca}^{2+}$ release (Fig. 4b and 4c). Although the two shRNAs had different 285 CLCC1 knockdown efficiencies, they impaired the ATP-induced $\mathrm{Ca}^{2+}$ amplitude 286 and rate to a similar extent. knockdown impaired ATP-induced $\mathrm{Ca}^{2+}$ oscillation (Extended Data Fig. 8a and 8b). Whereas less than $10 \%$ of cells exhibited only one ATP-induced $\mathrm{Ca}^{2+}$ spike in mock control or scrambled shRNA groups, the proportion was more than a half in the CLCC1 knockdown group. The impairment of ATP-induced $\mathrm{Ca}^{2+}$ release seems not to be caused by shRNA off-target effects, because the reexpression of full-length (WT) mCLCC1 restored the release damaged by $\mathrm{H} 3$ shRNA alone (Fig. $4 d$ and $4 e)$. In contrast, expression of mutant mCLCC1 lacking the ER lumen resident $2^{\text {nd }}$ loop ( $\Delta 2^{\text {nd }}$ loop) did not, suggesting the $2^{\text {nd }}$ loop of CLCC1 is crucial 296 for its functions. Interestingly, both ALS-associated S263R and W267R 297 mutations located in the $2^{\text {nd }}$ loop also significantly affected the internal $\mathrm{Ca}^{2+}$ release 298 relative to wildtype hCLCC1 (hWT) (Fig. 4f), further confirming the damaging effect 299 of the mutations on channel function.

$300 \quad$ Next, we asked whether CLCC1 regulates internal $\mathrm{Ca}^{2+}$ release through RyRs 301 (ryanodine receptors), the predominant intracellular $\mathrm{Ca}^{2+}$ channels expressed in 
302 cardiomyocytes ${ }^{10}$. To this end, we stimulated cardiomyocytes cultured from 303 wildtype $(+/+)$ and NM2453 mutant (NM/NM) mice with caffeine, an agonist for

304 RyR-mediated $\mathrm{Ca}^{2+}$ release. RyR-mediated $\mathrm{Ca}^{2+}$ release was significantly 305 reduced in NM/NM cardiomyocytes as compared to +/+ controls (Extended Data 306 Fig. 8c), demonstrating that CLCC1 facilitates $\mathrm{ER} \mathrm{Ca}^{2+}$ efflux through regulation of 307 the release process per se rather than regulation of a particular type of $\mathrm{Ca}^{2+}$ 308 release channels.

310 To examine whether the impaired $\mathrm{Ca}^{2+}$ release upon CLCC1 knockdown results

311 from a reduction in $\mathrm{ER} \mathrm{Ca}^{2+}$ load, we depleted the $\mathrm{ER} \mathrm{Ca}^{2+}$ store with cyclopiazonic 312 acid (CPA), an inhibitor of sarco/endoplasmic reticulum $\mathrm{Ca}^{2+}$-ATPase (SERCA) ${ }^{31}$.

313 Knockdown of CLCC1 by $\mathrm{H} 3$ but not $\mathrm{H} 4$ shRNA significantly reduced CPA314 sensitive cytosolic $\mathrm{Ca}^{2+}$ rise (Fig. 4g-4i), suggesting that impairment of $\mathrm{ER} \mathrm{Ca}^{2+}$ 315 content depends on CLCC1 dosage as H3 has higher knockdown efficiency than 316 H4 shRNA (Fig. 3e).

317 Given that depletion of CLCC1 increases ER volume (Fig. $3 g$ and 3h), we next 318 asked whether $\left[\mathrm{Ca}^{2+}\right]_{\mathrm{ER}}$ is also impaired. We employed a previously reported low 319 affinity $\mathrm{Ca}^{2+}$ probe, ER-GCaMP6-210 32 , which correctly responded to CPA320 induced internal $\mathrm{Ca}^{2+}$ depletion and follow-up ionomycin-mediated extracellular $321 \mathrm{Ca}^{2+}$ replenish (Extended Data Fig. 9). Compared to mock and scrambled 
322 shRNA controls, knockdown of CLCC1 by both H3 and H4 shRNAs significantly

323 decreased steady state [ $\left.\mathrm{Ca}^{2+}\right]_{\mathrm{ER}}$ level in cells expressing ER-GCaMP6-210 (Fig. 4j

324 and $4 \mathrm{k}$ ). The impairment caused by $\mathrm{H} 3$ shRNA was more severe than that by $\mathrm{H} 4$

325 shRNA, suggesting that depletion of CLCC1 decreases steady state $\left[\mathrm{Ca}^{2+}\right]_{\mathrm{ER}}$ level

326 in a dosage-dependent manner.

327 A conserved lysine (K298) is responsible for PIP2 facilitation of CLCC1 328 channel activity

329 As a necessary cofactor of many ion channels, PIP2, an acidic phospholipid of the 330 cell membrane, has been implicated in the regulation of ion channel functions, 331 including of intercellular cation channels ${ }^{33-35}$. To examine whether PIP2 affects 332 CLCC1 channel activity, we included 2\% PIP2 in the planar phospholipid bilayer. 333 Interestingly, PIP2 significantly increased the slope conductance $(80.1 \pm 2.5 \mathrm{pS})$ 334 and the open probability $\left(P_{\mathrm{o}}\right)$ of wildtype mCLCC1 (Fig. 5a and 5b). Given that 335 PIP2 regulates ion channels by binding to certain positively charged residues in 336 the channel protein ${ }^{34,35}$, we looked for positively charged residue(s) in CLCC1 and 337 a positively charged lysine (K298) drew our attention (Fig. 5c). It lies in a 338 consecutive row of six conserved residues-VPPTKA in the $2^{\text {nd }}$ loop, which is 339 required for $\mathrm{CLCC} 1$ facilitation of internal $\mathrm{Ca}^{2+}$ release (Fig. $4 \mathrm{~d}$ and $4 \mathrm{e}$ ). In 340 addition, K298 is downstream of two proline residues, which usually present strong 341 conformational rigidity, and lies at the beginning of a predicted alpha-helix. 
We expressed and purified K298A mutant mCLCC1 and incorporated it into the lipid bilayer in the absence of PIP2, the mutant protein exhibited single channel

344 activity with a slope conductance of $31.8 \pm 0.7 \mathrm{pS}$, slightly lower than that of wildtype mCLCC1 $(39.9 \pm 1.0 \mathrm{pS})($ Fig. $5 \mathrm{a}, 5 \mathrm{~d}$, and $5 \mathrm{f})$. The $P_{\mathrm{o}}$ at $0 \mathrm{mV}$ did not differ from that of wildtype mCLCC1 (Fig. 5a and 5f). Next, we mutated K298 to

347 the negatively charged residue glutamate (K298E). Like K298A, K298E also has 348 little effect on the channel activity in absence of PIP2 (Fig. 5f). However, unlike wildtype mCLCC1 responsible to PIP2 (Fig. 5b and 5e), both K298A and K298E mutants abolished the responses, in terms of conductance and $P_{\mathrm{o}}$ (Fig. 5e and 5f).

351 Therefore, we conclude that PIP2 facilitates CLCC1 channel activity and a 352 conserved K298 in the $2^{\text {nd }}$ loop is responsible for the facilitation.

In comparison to the K298A mutant protein in the lipid bilayer assay, proteins with the ALS patient mutations showed slope conductance that were significantly 355 lower than that of hWT in presence of PIP2 (Extended Data Fig. 10a-10c). 356 However, $P_{\mathrm{o}}$ of S263R and W267R did not differ from that of hWT in absence and 357 presence of PIP2, respectively (Extended Data Fig. 10d). Like hWT, S263R and 358 W267R mutant CLCC1 were in response to PIP2 (Extended Data Fig. 10c). 359 Therefore, we conclude that S263R and W267R located in the $2^{\text {nd }}$ loop and close 360 to K298 impair CLCC1 channel activity not through disruption of the PIP2-mediated 361 facilitation. 


\section{K298 is crucial for CLCC1 regulation of internal $\mathrm{Ca}^{2+}$ release}

363 If K298 is functionally important for CLCC1 channel activity, we wondered whether

$364 \mathrm{~K} 298$ is equally important for internal $\mathrm{Ca}^{2+}$ release. To examine this, we

365 employed a lentiviral inducible system to stably express wildtype and K298A 366 mutant mCLCC1 in 293FT cells in a controllable manner (Extended Data Fig. 11a).

367 Expression of exogenous mCLCC1 proteins was induced after application of 368 doxycycline (Dox) (Extended Data Fig. 11b). Both the exogenous wildtype and 369 K298A mutant mCLCC1 interacted with the endogenous hCLCC1 (Extended Data 370 Fig. 11c), as shown by co-immunoprecipitation, supporting complex formation by 371 exogenous mCLCC1 and endogenous hCLCC1 (Extended Data Fig. 4b). 372 Induction of wildtype mCLCC1 did not alter the amplitude and rate of ATP-induced $373 \mathrm{Ca}^{2+}$ release (Fig. 5g-5i). However, expression of K298A mutant mCLCC1 374 significantly suppressed such activities, as shown by the reduction in both the 375 amplitude and rate when compared to un-induced (minus Dox) cells or cells 376 induced to express wildtype mCLCC1. In addition, induction of K298A mutant 377 mCLCC1 expression, but not wildtype mCLCC1, decreased the number of ATP378 induced $\mathrm{Ca}^{2+}$ oscillation (Extended Data Fig. 11d and 11e). These findings are 379 all similar to that found in CLCC1-knockdown cells (Fig. 4a-4c and Extended Data 380 Fig. $8 a$ and $8 b)$, suggesting a dominant-negative effect of the mutant protein in 381 CLCC1 channel function. Taken together, our findings reveal that a conserved 
$382 \mathrm{~K} 298$ in the $2^{\text {nd }}$ loop is functionally important for CLCC1 to regulate the internal

$383 \mathrm{Ca}^{2+}$ release.

K298A mutation promotes motor neuron loss and enlarges ER volume in vivo

386 To examine the in vivo effect of mutating the conserved K298 residue, as it is 387 critical for PIP2 facilitation on CLCC1 channel activity and internal $\mathrm{Ca}^{2+}$ release, 388 we generated K298A knock-in mouse (Extended Data Fig. 12a and 12b). 389 Although expression of K298A mutant mRNA and protein was confirmed by 390 Sanger sequencing and mass spectrometry (Extended Data Fig. 12c-12e), the 391 expression level of K298A mutant protein was as low as that of the NM2453 allele 392 (Fig. 5j), reminiscent of ALS-associated S263R and W267R mutant CLCC1 (Fig. 393 1f). Like Clcc1 KO (Supplementary Table 2), we failed to produce mouse 394 homozygous for K298A (Supplementary Table 3), indicating that K298 is a key 395 residue for CLCC1 expression and its essential function in vivo.

397 were viable but displayed severe body weight loss, hind leg weakness, trunk 398 shaking, tail flagging, abnormal gaits, and ataxia phenotypes as early as 3 months 399 of age (Supplementary Movie 1), much earlier than the phenotype onset shown in 400 the NM/NM mice (> 12 month of age) ${ }^{18}$. Like NM/NM mice, the compound 401 heterozygotes displayed ER stress (Fig. 5k) and neuron degeneration in cerebellar 
402 granule neurons (Extended Data Fig. 12f). ER stress was also evidenced in

403 hippocampal granule neurons in the compound heterozygotes but not in NM/NM 404 mice (Extended Data Fig. 12g). As rare CLCC1 mutations were found in ALS 405 (Fig. 1) and the severe motor impairment and hind leg muscle weakness were 406 shown in NM/K298A mice (Supplementary Movie 1), we next examined motor 407 neuron pathologies in these compound heterozygotes mice. Indeed, ubiquitin408 positive inclusions in ChAT-positive motor neurons and their number loss, two key 409 ALS pathologies, were evidenced in the mutant spinal cords (Fig. $5 \mathrm{I}$ and $5 \mathrm{~m}$ ), 410 supporting CLCC1 is a potential ALS-causing gene. As knockdown of CLCC1 impairs ER ion homeostasis and leads to ER swelling 412 (Fig. 3), we next asked whether dysfunction of CLCC1 impairs ER morphology in 413 vivo. To this end, we examined the cerebella from wildtype and K298A/NM mice 414 by TEM. We observed that instead of ribosome-bound and tubule-like ER 415 morphologies observed in wildtype cerebellar granule neurons, the mutant 416 neurons harbored enlarged, stubby, and less ribosome-bound ER (Fig. 5n). 417 Indeed, the ER width of mutant granule neurons was significantly increased 418 compared to that of wildtype (Fig. 5o). Taken together, our findings demonstrate 419 that disruption of channel function by the K298A promotes ER stress and motor 420 neuron loss and enlarges ER volume in the diseased neuron in vivo. 
421 Increased penetrance of K298A allele and cell-autonomous effect of CLCC1

422 loss in motor neuron loss

423 In the $\mathrm{K} 298 \mathrm{~A} /+$ colony, we were surprised to find that a few $\left(12 / 182, \mathrm{~K} 298 \mathrm{~A} /+^{*}\right)$

424 animals appeared to exhibit severe phenotypes as early as postnatal $90.9 \pm 5.5$

425 days (Fig. 6a-6c and Supplementary Movie 2), reminiscent of the phenotypes

426 shown in NM/K298A (Fig. 5 and Supplementary Movie 1). Because dosage of

427 CLCC1 is critical for the mutant phenotypes, we examined CLCC1 expression in 428 various tissues in these $\mathrm{K} 298 /+^{*}$ animals. As expected, CLCC1 expression level

429 was significantly decreased in these tissues compared to that of wildtype and $430 \mathrm{~K} 298 \mathrm{~A} /+$ animals (Fig. 6d and Extended Data Fig. 13a). The decreased CLCC1 431 expression seems not to be explained by the decreased C/cc1 mRNA (Extended 432 Data Fig. 13b). Because ALS-associated mutations we identified appear 433 dominant, increased disease penetrance of K298A heterozygous mutant CLCC1 434 weights the physiological relevance to ALS.

To gain insight into cell-autonomous or non-cell-autonomous effect of Clcc1 436 loss-of-function in motor neuron degeneration, we generated Clcc1 floxed (fl) 437 mouse (Fig. 6e) and crossed it to ChAT-Cre mouse ${ }^{36}$, to knockout Clcc1 in ChAT438 positive motor neuron in spinal cord. ER stress was evidenced by upregulation 439 of both Bip and ERp72 in ChAT-positive motor neurons in ChAT-Cre/+;fl/fl but not 440 ChAT-Cre/+;fl/+ spinal cords (Fig. $6 f$ and 6g). Misfolded protein accumulation 
441 was also evidenced by upregulation of ubiquitin in these Clcc1 conditional KO 442 neurons (Fig. 6f and 6g). Compared to nucleus-localized TDP-43 in ChAT443 Cre/+;fl/+ motor neurons, cytoplasm-mislocalized and ubiquitin-positive TDP-43 444 (Fig. 6h), one of the pathological hallmarks of ALS ${ }^{37-39}$, were documented in the 445 conditional KO neurons. Indeed, all the ChAT-Cre/+;fl/fl animals died before P30 446 (Fig. 6i) with significant loss of motor neurons (Fig. 6j). Therefore, we conclude 447 that the effect of Clcc1 loss-of-function in motor neuron loss is cell-autonomous. 448 
Discussion:

Here, we characterized CLCC1 as a pore-forming component of an ER anion channel, activity of which is inhibited by luminal $\mathrm{Ca}^{2+}$ but facilitated by PIP2. We link rare CLCC1 mutations to ALS and demonstrate that the ALS-associated mutations impair CLCC1 channel activity, damage ER ion homeostasis, and promote ER stress in brain, implying that disruption of ER ion homeostasis maintained by CLCC1 underlies etiology of ALS.

In the ALS Chinese cohort, S263R was found in two unrelated patients, 457 suggesting it is a potential disease-causing mutation. Physically, S263 and W267 458 are in close proximity (Fig. 1d). Functionally, both S263R and W267R lead to the 459 biological consequences to a similar extent (Fig. 1e, 1f, 2f, 3d, and 4f), suggesting 460 they impair CLCC1 channel function probably through a similar mechanism. Like 461 ALS-associated S263R and W267R mutations, K298A leads to similar phenotypes 462 (Fig. 5), supporting the notion that impairment of ER ion homeostasis maintained 463 by CLCC1 leads to neurodegeneration. Phenotypic analysis from five Clcc1 464 alleles, including the two ALS alleles we generated, revealed that severity of 465 CLCC1 mutant phenotypes is truly dose-dependent in vivo (Extended Data Fig. 466 14), which are evidenced in vitro by the effects of CLCC1 knockdown dosage467 dependently impairing ER volume, $\mathrm{ER} \mathrm{Ca}^{2+}$ content, and steady state $\left[\mathrm{Ca}^{2+}\right]_{\mathrm{ER}}$ (Fig. 4683 and 4 ). Given that CLCC1 is ubiquitously expressed ${ }^{18}$, CLCC1 ER functions 
described here, including maintenance of steady-state $\left[\mathrm{Cl}^{-}\right]_{\mathrm{ER}}$ and morphology and regulation of ER $\mathrm{Ca}^{2+}$ homeostasis, could be applied to non-neuronal tissues and cell types.

During internal $\mathrm{Ca}^{2+}$ release, both $\mathrm{Cl}^{-}$efflux through $\mathrm{ER}$ anion channel(s) and $\mathrm{K}^{+}$influx through either TRIC family channels ${ }^{12}$ or RyRs/IP3Rs ${ }^{8,15,40}$ are indispensable for neutralization of membrane charge and balance of luminal osmolarity at the same time (Extended Data Fig. 15). Upon depletion/dysfunction of CLCC1, ER Ca ${ }^{2+}$ release was impaired (Fig. 4) and we speculate that it was caused by $\mathrm{Cl}^{-}$through $\mathrm{CLCC} 1$ no longer compensating for the membrane charge. Therefore, double the amount of $\mathrm{K}^{+}$influx through ER cation channels was needed to partially neutralize the membrane potential induced by $\mathrm{Ca}^{2+}$ release, which in turn increases luminal osmolarity. In addition to this acute effect, CLCC1 maintains ER anion homeostasis and depletion of CLCC1 increases [ $\mathrm{Cl}]_{\mathrm{ER}}$ (Fig. 3), which even worsens luminal osmolarity and ER swelling (Extended Data Fig. 15). Decreased CLCC1 dosage-dependently lowers ER Ca ${ }^{2+}$ content (Fig. 4g-4i), which together with increased ER volume further decrease steady state $\left[\mathrm{Ca}^{2+}\right]_{E R}$ (Fig. $4 \mathrm{j}$ and $4 \mathrm{k}$ ) and damage $\mathrm{Ca}^{2+}$-dependent protein folding capability, eventually leading to ER stress, misfolded protein accumulation, and neurodegeneration ${ }^{41}$ (Extended Data Fig. 15). 
CLCC1 shares little sequence similarity with any known ion channel, indicating that it belongs to a new channel family, therefore, we suggest renaming it ER anion channel 1 (ERAC1). Luminal $\mathrm{Ca}^{2+}$ inhibition on the channel activity prompts us to speculate a pre-inhibition mechanism in the resting state. However, when $\mathrm{Ca}^{2+}$ releases from ER, the local luminal $\left[\mathrm{Ca}^{2+}\right]$ drops sharply, which in turn relieves the inhibition (Extended Data Fig. 15). CLCC1 channel activity is facilitated by PIP2 (Fig. 5), reminiscent of PIP2 positive regulation of TRIC channel activity ${ }^{34}$. Amplification of both CLCC1 and TRIC channel conductance by PIP2 may have biological relevance to large $\mathrm{Ca}^{2+}$ conductance of $\mathrm{RyRs} / \mathrm{IP} 3 \mathrm{Rs}$ during internal $\mathrm{Ca}^{2+}$ release ${ }^{8,40}$. CLCC1 channel activity is insensitive to voltage (Fig. 2d) but sensitive to DIDS (Extended Data Fig. 6b and 6c), reminiscent of some early reported chloride currents recorded from SR/ER membrane preparation ${ }^{13,42-44}$, but different from previously described $\mathrm{CLCC} 1$ currents ${ }^{19}$. Purified C350F mutant CLCC1 restored both MTSET- and DIDS-mediated cytoplasmic side modulation on channel activity (Fig. $2 \mathrm{~h}$ and Extended Data Fig. $6 \mathrm{~b}$ and $6 \mathrm{c}$ ), suggesting that C350 that lies at the end of predicated TM3 is close to CLCC1 anion permeation pathway.

In K298A/+ mouse colony, K298A heterozygous mutation increased penetrance in disease progression by affecting expression of both mutant and wildtype CLCC1 (Extended Data Fig. 13), which form protein complex (Extended 
508 Data Fig. 11c). Decreased CLCC1 is likely mediated by ER-associated 509 degradation (ERAD) pathway, a cellular mechanism for ER protein quality control 51045,46 . Cell-autonomous effect of C/cc1 loss-of-function on ubiquitin-positive and 511 mislocalized TDP-43 (Fig. 6) links CLCC1 dysfunction to a common ALS pathology 512 and its underlying disease mechanisms ${ }^{37,47,48}$. Dysfunction of RNA binding 513 proteins (RBPs), including TDP-43, often leads to stress granule processing ${ }^{38,39}$. 514 It will be intriguing to further investigate the crosstalk between ER and 515 membraneless organelles, like stress granule ${ }^{49}$, and how these dysfunctions in 516 two cellular systems converge with the pathogenesis of ALS. 
534 Development" (Strategic Priority Research Program of the Chinese Academy of

535 Sciences, Grant No. XDA12040221 and XDA15050308), the National Natural

536 Science Foundation of China $(81974197,81371361,31571097,81773707$, 
$53782071426,81873784,61327014,92049114$ and 61175103), and the Shanghai

538 Science and Technology Innovation Fund 15431901500.

\section{Author contributions:}

540 L.G. generated the CLCC1 N- and C-terminal antibodies, performed the

541 biochemical experiments, immunofluorescence staining, and data analysis, and

542 characterized the K298A, S263R and W267R knock-in mouse lines. Q.M.

543 conducted planar phospholipid bilayer recording and data analysis. L.G., X.L.,

544 and B.X. performed calcium imaging. L.L. and X.P. generated the K298A, S263R,

545 and W267R knock-in mouse lines. L.L. isolated the primary cardiomyocyte. J.H.

546 and D.F. collected ALS sample and performed patient diagnosis and exome-

547 sequencing. L.G., J.H., Z.G., and Y.J. wrote the manuscript. D.F., Z.G., and Y.J

548 designed and supervised experiments.

549 Declaration of Interests:

550 The authors declare no competing interests. 
552 References:

5531 Cutting, G. R. Cystic fibrosis genetics: from molecular understanding to clinical application. Nat

$554 \quad$ Rev Genet 16, 45-56, doi:10.1038/nrg3849 (2015).

5552 Duran, C., Thompson, C. H., Xiao, Q. \& Hartzell, H. C. Chloride channels: often enigmatic, rarely

556 predictable. Annu Rev Physiol 72, 95-121, doi:10.1146/annurev-physiol-021909-135811 (2010).

5573 Jentsch, T. J. \& Pusch, M. CLC Chloride Channels and Transporters: Structure, Function,

$558 \quad$ Physiology, and Disease. Physiol Rev 98, 1493-1590, doi:10.1152/physrev.00047.2017 (2018).

5594 Jentsch, T. J., Stein, V., Weinreich, F. \& Zdebik, A. A. Molecular structure and physiological

560 function of chloride channels. Physiol Rev 82, 503-568, doi:10.1152/physrev.00029.2001 (2002).

5615 Stauber, T. \& Jentsch, T. J. Chloride in vesicular trafficking and function. Annu Rev Physiol 75,

562 453-477, doi:10.1146/annurev-physiol-030212-183702 (2013).

5636 Argenzio, E. \& Moolenaar, W. H. Emerging biological roles of Cl- intracellular channel proteins.

$564 \quad J$ Cell Sci 129, 4165-4174, doi:10.1242/jcs.189795 (2016).

5657 Gibson, A. et al. hCLCA1 and mCLCA3 are secreted non-integral membrane proteins and

566 therefore are not ion channels. J Biol Chem 280, 27205-27212, doi:10.1074/jbc.M504654200

567 (2005).

5688 Fill, M. \& Copello, J. A. Ryanodine receptor calcium release channels. Physiol Rev 82, 893-922,

569 doi:10.1152/physrev.00013.2002 (2002).

5709 Clapham, D. E. Calcium signaling. Cell 131, 1047-1058, doi:10.1016/j.cell.2007.11.028 (2007).

$571 \quad 10 \quad$ Berridge, M. J. The endoplasmic reticulum: a multifunctional signaling organelle. Cell Calcium

$572 \quad 32,235-249$ (2002).

57311 Takeshima, H., Venturi, E. \& Sitsapesan, R. New and notable ion-channels in the

574 sarcoplasmic/endoplasmic reticulum: do they support the process of intracellular Ca2+ release? $J$

$575 \quad$ Physiol, doi:10.1113/jphysiol.2014.281881 (2014).

57612 Yazawa, M. et al. TRIC channels are essential for $\mathrm{Ca} 2+$ handling in intracellular stores. Nature

$577 \quad 448,78-82$, doi:10.1038/nature05928 (2007).

57813 Miller, C. Voltage-gated cation conductance channel from fragmented sarcoplasmic reticulum:

$579 \quad$ steady-state electrical properties. J Membr Biol 40, 1-23 (1978).

$58014 \quad$ Edwards, J. C. \& Kahl, C. R. Chloride channels of intracellular membranes. FEBS Lett 584, 2102-

$581 \quad$ 2111, doi:10.1016/j.febslet.2010.01.037 (2010).

58215 Gillespie, D. \& Fill, M. Intracellular calcium release channels mediate their own countercurrent:

583 the ryanodine receptor case study. Biophys $J$ 95, 3706-3714, doi:10.1529/biophysj.108.131987

$584 \quad$ (2008).

$58516 \quad$ Zsolnay, V., Fill, M. \& Gillespie, D. Sarcoplasmic Reticulum Ca(2+) Release Uses a Cascading

$586 \quad$ Network of Intra-SR and Channel Countercurrents. Biophys $J$ 114, 462-473,

$587 \quad$ doi:10.1016/j.bpj.2017.11.3775 (2018).

58817 al-Awqati, Q. Chloride channels of intracellular organelles. Curr Opin Cell Biol 7, 504-508

$589 \quad$ (1995). 


\begin{tabular}{|c|c|c|}
\hline 590 & 18 & ia, Y., Jucius, T. J., Cook, S. A. \& Ackerman, S. L. Loss of Clcc1 results in ER stress, misfolded \\
\hline 591 & & protein accumulation, and neurodegeneration. $J$ Neurosci 35, 3001-3009, \\
\hline 2 & & doi:10.1523/JNEUROSCI.3678-14.2015 (2015). \\
\hline 3 & 19 & Nagasawa, M., Kanzaki, M., Iino, Y., Morishita, Y. \& Kojima, I. Identification of a novel chloride \\
\hline 594 & & channel expressed in the endoplasmic reticulum, golgi apparatus, and nucleus. $J$ Biol Chem $\mathbf{2 7 6}$, \\
\hline 95 & & 20413-20418, doi:10.1074/jbc.M100366200 (2001). \\
\hline 596 & 20 & Lindholm, D., Wootz, H. \& Korhonen, L. ER stress and neurodegenerative diseases. Cell Death \\
\hline 597 & & Differ 13, 385-392, doi:10.1038/sj.cdd.4401778 (2006). \\
\hline 598 & 21 & Cook, C. \& Petrucelli, L. Genetic Convergence Brings Clarity to the Enigmatic Red Line in ALS. \\
\hline 599 & & Neuron 101, 1057-1069, doi:10.1016/j.neuron.2019.02.032 (2019). \\
\hline 600 & 22 & Taylor, J. P., Brown, R. H., Jr. \& Cleveland, D. W. Decoding ALS: from genes to mechanism. \\
\hline 601 & & Nature 539, 197-206, doi:10.1038/nature20413 (2016). \\
\hline 602 & 23 & Farhan, S. M. K. et al. Exome sequencing in amyotrophic lateral sclerosis implicates a novel gene, \\
\hline 03 & & DNAJC7, encoding a heat-shock protein. Nat Neurosci 22, 1966-1974, doi:10.1038/s41593-019- \\
\hline 604 & & 0530-0 (2019). \\
\hline 605 & 24 & Vasilescu, J., Guo, X. \& Kast, J. Identification of protein-protein interactions u \\
\hline 606 & & linking and mass spectrometry. Proteomics 4, 3845-3854, doi:10.1002/pmic.200400856 (2004). \\
\hline 607 & 25 & Suski, J. M. et al. Isolation of plasma membrane-associated membranes from rat \\
\hline 608 & & 9, 312-322, doi:10.1038/nprot.2014.016 (2014). \\
\hline 609 & 26 & Akabas, M. H., Stauffer, D. A., Xu, M. \& Karlin, A. Acetylcholine receptor channel structure \\
\hline 610 & & probed in cysteine-substitution mutants. Science 258, 307-310 (1992). \\
\hline 611 & 27 & Matulef, K. \& Maduke, M. Side-dependent inhibition of a prokaryotic ClC by DIDS. Biophys $J$ \\
\hline 612 & & 89, 1721-1730, doi:10.1529/biophysj.105.066522 (2005). \\
\hline 613 & 28 & Zhong, S., Navaratnam, D. \& Santos-Sacchi, J. A genetically-encoded YFP sensor with enhanced \\
\hline 614 & & chloride sensitivity, photostability and reduced ph interference demonstrates augmented \\
\hline 615 & & transmembrane chloride movement by gerbil prestin (SLC26a5). PLoS One 9, e99095, \\
\hline 616 & & doi:10.1371/journal.pone.0099095 (2014). \\
\hline 617 & 29 & Shcherbakova, D. M. \& Verkhusha, V. V. Near-infrared fluorescent proteins for multicolor in vivo \\
\hline 18 & & imaging. Nat Methods 10, 751-754, doi:10.1038/nmeth.2521 (2013). \\
\hline 619 & 30 & Jentsch, T. J. VRACs and other ion channels and transporters in the regulation of cell volume and \\
\hline 20 & & beyond. Nat Rev Mol Cell Biol 17, 293-307, doi:10.1038/nrm.2016.29 (2016). \\
\hline 21 & 31 & Seidler, N. W., Jona, I., Vegh, M. \& Martonosi, A. Cyclopiazonic acid is a specific inhibitor of the \\
\hline 622 & & Ca2+-ATPase of sarcoplasmic reticulum. J Biol Chem 264, 17816-17823 (1989). \\
\hline 623 & 32 & de Juan-Sanz, J. et al. Axonal Endoplasmic Reticulum $\mathrm{Ca}(2+)$ Content Controls Release \\
\hline 624 & & Probability in CNS Nerve Terminals. Neuron 93, 867-881 e866, doi:10.1016/j.neuron.2017.01.010 \\
\hline 625 & & (2017). \\
\hline 626 & 33 & Suh, B. C. \& Hille, B. Regulation of ion channels by phosphatidylinositol 4,5-bisphosphate. Curr \\
\hline 627 & & Opin Neurobiol 15, 370-378, doi:10.1016/j.conb.2005.05.005 (2005). \\
\hline 628 & 34 & Yang, H. et al. Pore architecture of TRIC channels and insights into their gating mechanism. \\
\hline 29 & & Nature 538, 537-541, doi:10.1038/nature19767 (2016). \\
\hline
\end{tabular}




\begin{tabular}{|c|c|c|}
\hline $\begin{array}{l}630 \\
631\end{array}$ & 35 & $\begin{array}{l}\text { Suh, B. C. \& Hille, B. PIP2 is a necessary cofactor for ion channel function: how and why? Annu } \\
\text { Rev Biophys 37, 175-195, doi:10.1146/annurev.biophys.37.032807.125859 (2008). }\end{array}$ \\
\hline $\begin{array}{l}632 \\
633\end{array}$ & 36 & $\begin{array}{l}\text { Rossi, J. et al. Melanocortin-4 receptors expressed by cholinergic neurons regulate energy balance } \\
\text { and glucose homeostasis. Cell Metab 13, 195-204, doi:10.1016/j.cmet.2011.01.010 (2011). }\end{array}$ \\
\hline 634 & 37 & Lagier-Tourenne, C., Polymenidou, M. \& Cleveland, D. W. TDP-43 and FUS/TLS: emerging \\
\hline 635 & & roles in RNA processing and neurodegeneration. Hum Mol Genet 19, R46-64, \\
\hline 636 & & doi:10.1093/hmg/ddq137 (2010). \\
\hline 637 & 38 & Renton, A. E., Chio, A. \& Traynor, B. J. State of play in amyotrophic lateral sclerosis genetics. \\
\hline 638 & & Nat Neurosci 17, 17-23, doi:10.1038/nn.3584 (2014). \\
\hline 639 & 39 & Ling, S. C., Polymenidou, M. \& Cleveland, D. W. Converging mechanisms in ALS and FTD: \\
\hline 640 & & disrupted RNA and protein homeostasis. Neuron 79, 416-438, doi:10.1016/j.neuron.2013.07.033 \\
\hline 641 & & (2013). \\
\hline 642 & 40 & Foskett, J. K., White, C., Cheung, K. H. \& Mak, D. O. Inositol trisphosphate receptor Ca2+ \\
\hline 643 & & release channels. Physiol Rev 87, 593-658, doi:10.1152/physrev.00035.2006 (2007). \\
\hline 644 & 41 & Braakman, I. \& Bulleid, N. J. Protein folding and modification in the mammalian endoplasmic \\
\hline 645 & & reticulum. Аnпи Rev Biochem 80, 71-99, doi:10.1146/annurev-biochem-062209-093836 (2011). \\
\hline 646 & 42 & Ran, S., Fuller, C. M., Arrate, M. P., Latorre, R. \& Benos, D. J. Functional reconstitution of a \\
\hline 647 & & chloride channel protein from bovine trachea. J Biol Chem 267, 20630-20637 (1992). \\
\hline 648 & 43 & Kourie, J. I., Laver, D. R., Junankar, P. R., Gage, P. W. \& Dulhunty, A. F. Characteristics of two \\
\hline 649 & & types of chloride channel in sarcoplasmic reticulum vesicles from rabbit skeletal muscle. Biophys \\
\hline 650 & & $J$ 70, 202-221, doi:10.1016/S0006-3495(96)79564-4 (1996). \\
\hline 651 & 44 & Tanifuji, M., Sokabe, M. \& Kasai, M. An anion channel of sarcoplasmic reticulum incorporated \\
\hline 652 & & into planar lipid bilayers: single-channel behavior and conductance properties. $J$ Membr Biol $\mathbf{9 9}$, \\
\hline 653 & & 103-111 (1987). \\
\hline 654 & 45 & Trombetta, E. S. \& Parodi, A. J. Quality control and protein folding in the secretory pathway. \\
\hline 655 & & Annu Rev Cell Dev Biol 19, 649-676, doi:10.1146/annurev.cellbio.19.110701.153949 (2003). \\
\hline 656 & 46 & Vembar, S. S. \& Brodsky, J. L. One step at a time: endoplasmic reticulum-associated degradation. \\
\hline 657 & & Nat Rev Mol Cell Biol 9, 944-957, doi:10.1038/nrm2546 (2008). \\
\hline 658 & 47 & Neumann, M. et al. Ubiquitinated TDP-43 in frontotemporal lobar degeneration and amyotrophic \\
\hline 659 & & lateral sclerosis. Science 314, 130-133, doi:10.1126/science.1134108 (2006). \\
\hline 660 & 48 & Dugger, B. N. \& Dickson, D. W. Pathology of Neurodegenerative Diseases. Cold Spring Harb \\
\hline 661 & & Perspect Biol 9, doi:10.1101/cshperspect.a028035 (2017). \\
\hline 662 & 49 & Lee, J. E., Cathey, P. I., Wu, H., Parker, R. \& Voeltz, G. K. Endoplasmic reticulum contact sites \\
\hline 663 & & regulate the dynamics of membraneless organelles. Science 367 , doi: $10.1126 /$ science.aay 7108 \\
\hline & & (2020). \\
\hline
\end{tabular}

665

666 
667

668

669

670

671

672

673

674

675

676

677

678

679

680

681

682

683

684

685

686

\section{Materials and methods:}

\section{Protein expression and purification.}

The DNA fragments encoding mouse CLCC1-N (residues 12-200, NM_145543.2) and CLCC1-C (residues 355-539) were cloned into pET28A (Novagen) with an Nterminal $6 \times$ His tag or into pMAL-cRI with an N-terminal MBP (maltose binding protein, NEB) tag. The recombinant CLCC1 were expressed in BL21 derivative Rosetta (DE3) at $37{ }^{\circ} \mathrm{C}$ overnight. After ultrasonic cell disruption, the recombinant proteins in the soluble fractions were purified by Ni-NTA resin (Qiagen) or amylose resin (NEB) and dialyzed overnight in $10 \mathrm{mM}$ PBS solution. For insect expression system, the full-length mouse and human CLCC1 (wildtype, C350F, K298A, and K298E, S263R and W267R) were cloned into pFastbac-1 (Invitrogen) with a C-terminal His 10 tag. The bacmids were extracted from $\mathrm{DH} 10$ Bac bacteria and transfected into Sf9 insect cells, which were grown in SFX-Insect cell culture medium (GE Healthcare) at $26{ }^{\circ} \mathrm{C}$ to generate and amplify baculovirus (Bac-toBac system, Invitrogen). About $200 \mathrm{ml}$ of High Five insect cells $\left(1 \times 10^{6}\right.$ cells per ml SIM HF culture medium, Sino Biological Inc.) were infected by $4 \mathrm{ml}$ baculovirus to express the recombinant proteins. The infected High Five cells were harvested 48 hours after infection and homogenized in the TBS lysis buffer [50 mM Tris- $\mathrm{HCl}$

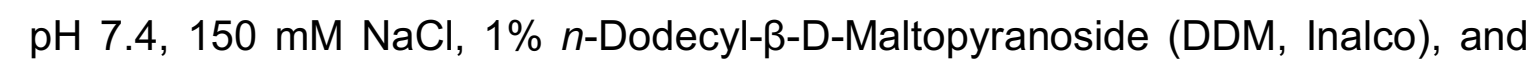
protease inhibitor cocktail, including $2 \mu \mathrm{g} / \mathrm{ml}$ pepstatin A, $4 \mu \mathrm{g} / \mathrm{ml}$ aprotinin, 10 
$687 \mathrm{mg} / \mathrm{ml}$ 4-(2-Aminoethyl) benzenesulfonyl fluoride hydrochloride, $4 \mu \mathrm{g} / \mathrm{ml}$ bestatin,

$6884 \mu \mathrm{g} / \mathrm{ml} \mathrm{E}-64,4 \mu \mathrm{g} / \mathrm{ml}$ leupeptin, and $1 \mathrm{mM}$ phenylmethane sulphonylfluoride] on

689 ice for 30 strokes with Dounce homogenizer, and then rotated for additional 30

690 minutes. The cell debris was removed by centrifugation at $30,000 \times \mathrm{g}$ for 1 hour.

691 The supernatant was harvested carefully, added $10 \mathrm{mM}$ imidazole, and incubated

692 with Ni-NTA resin (Qiagen). The resin was washed with TBS buffer containing

$6930.05 \%$ DDM and $100 \mathrm{mM}$ imidazole. The proteins were eluted from beads with

694 TBS buffer containing $0.05 \%$ DDM and $300 \mathrm{mM}$ imidazole. The resulting 695 proteins were treated with $2 \mathrm{mM} \mathrm{DTT}$ and incubated on ice for 30 minutes. The 696 final concentrated proteins were further purified by a size-exclusion 697 chromatography (Superose 6 Increase, GE Healthcare) in the TBS buffer 698 containing $0.025 \% \mathrm{DDM}$ and $2 \mathrm{mM}$ DTT. The positions of some standard 699 molecular weight markers shown in user manual (GE Healthcare) were used to 700 estimate the size of protein complex. The peak fractions were collected, frozen 701 in liquid nitrogen, and stored at $-80^{\circ} \mathrm{C}$ for electrophysiology studies.

702 Planar bilayer lipid membrane recording.

703 Lipid bilayers formed across an aperture $0.2 \mathrm{~mm}$ in diameter in a delrin cup, with 704 a mixture of phosphatidylcholine (PC), phosphatidylserine (PS) (Avanti Polar 705 Lipids) and phosphoethanolamine (PE) (Lipoid) in a weight ratio of 1:2:2. The 706 lipids were dissolved in $n$-decane (Sigma) at a concentration of $50 \mathrm{mg} \mathrm{lipid} / \mathrm{ml} n$ - 
707 decane. All solutions were buffered by 10 mM HEPES pH 7.4. The lipid bilayer 708 separated the cis $(\mathrm{In})$ solution from the trans (Ex) solution $(1.0 \mathrm{ml}$ each) and the 709 purified wildtype CLCC1 and its mutant variants were added to the cis side of a 710 lipid bilayer membrane. The purified proteins were added at cis side and the 711 membrane potential represents the voltage potential at trans side. The single

712 channel currents were recorded by adding $3.5 \mu \mathrm{l}$ of $1.8 \mathrm{mg} / \mathrm{ml}$ protein to the cis

713 side in asymmetric $\mathrm{KCl}$ solution $(\operatorname{In} / \mathrm{Ex}, 150 / 15 \mathrm{mM})$ at indicated voltages. The

714 macroscopic currents were recorded by adding $20.0 \mu \mathrm{l}$ of $1.8 \mathrm{mg} / \mathrm{ml}$ protein. The

715 membrane potentials were held at $+60 \mathrm{mV}$ and then stepped to a prepulse from -

$71640 \mathrm{mV}$ to $+100 \mathrm{mV}$ with $20 \mathrm{mV}$ increments for $3 \mathrm{~s}$ to elicit currents. The channel

717 currents were recorded in a voltage-clamp mode using a Warner BC-535 bilayer

718 clamp amplifier (Warner Instruments) filtered at $1 \mathrm{kHz}, 25^{\circ} \mathrm{C}$. The currents were

719 digitized using pCLAMP 10.4 software (Molecular Devices). The single-channel

720 conductance was determined by fitting to Gaussian functions. Opening times

721 less than $0.5-1.0 \mathrm{~ms}$ were ignored. The theoretical equilibrium potential was

722 calculated using the Nernst equation. The open probability $P_{\mathrm{o}}=\mathrm{t} / \mathrm{T}$, where $\mathrm{t}$ is

723 the total time that the channel is observed in the open state and $\mathrm{T}$ is the total

724 recording time. The ion selectivity was calculated using the Goldman-Hodgkin725 Katz flux equation. 
a. Monovalent anion: $E_{r e v}=-\frac{R T}{z F} \ln \frac{P_{\mathrm{A}}[\mathrm{A}]_{\circ}}{P_{\mathrm{B}}[\mathrm{B}]_{\mathrm{i}}}$

b. Divalent anion: $E_{\text {rev }}=-\frac{R T}{F} \ln \left(\sqrt{\frac{4 P_{\mathrm{SO} 4}[\mathrm{SO} 4]_{\mathrm{o}}}{P_{\mathrm{Cl}}[\mathrm{Cl}]_{\mathrm{i}}}+\frac{1}{4}}-\frac{1}{2}\right)$

729 To examine the inhibitory effects of [2-(trimethylammonium)ethyl]

730 methanethiosulfonate bromide (MTSET) and 4,4'-Diisothiocyano-2,2'-

731 stilbenedisulfonic acid (DIDS), a certain amount of stocks of the two drugs were

732 added into either cis or trans chamber using pipette.

733 The generation and purification of CLCC1 polyclonal antibodies.

734 To generate CLCC1 polyclonal antibodies, the purified mCLCC1-N (residues 12-

735 200) and mCLCC1-C (residues 355-539) tagged with MBP were used to immunize

736 the rabbits (SPF Japanese white rabbit). The subcutaneous inoculation was

737 given once two weeks at least 3 times $(0.1 \mathrm{mg}$ antigen in complete/incomplete

738 Freund's Adjuvant/rabbit, Sigma). The rabbit anti-serum was collected and

739 purified by NHS-activated Sefinose beads conjugated by His-tagged mCLCC1-N

740 or mCLCC1-C. The resulting antigen-antibody complexes were washed with

741 PBS containing $0.15 \%$ Triton $\mathrm{X}-100$ to reduce non-specific binding. The

742 polyclonal antibodies with high affinity were eluted from the Sefinose beads by 50

$743 \mathrm{mM}$ glycine $(\mathrm{pH} 2.5)$, and neutralized to $\mathrm{pH} 7.4$ immediately with Tris- $\mathrm{HCl}$ buffer.

744 Microsome isolation and protease digestion. 
745 The microsome isolation was performed as previously described with slight

746 modifications ${ }^{25}$. The brains and the livers of wildtype mice $(0.5 \mathrm{mg}$ tissue each

747 preparation) were disrupted by using Dounce homogenizer for 30 strokes in a 748 working buffer (225 mM mannitol, $75 \mathrm{mM}$ sucrose, and $30 \mathrm{mM}$ Tris- $\mathrm{HCl}, \mathrm{pH} 7.4$ )

749 on ice. The nuclei and unbroken cells were removed by centrifugation at $1,000 \times$

$750 \mathrm{~g}$ for 10 minutes. The supernatants containing the plasma membrane (PM) and

751 the endoplasmic reticulum (ER) fraction were harvested by a further centrifugation

752 at $10,000 \times \mathrm{g}$ for 10 minutes. The final pellet was collected at $25,000 \times \mathrm{g}$ for 30

753 minutes and resuspended in the working buffer. All centrifugation steps were

754 executed at $4{ }^{\circ} \mathrm{C}$. Protease digestion assay was performed as previously

755 reported with some modifications (PMID: 20826464). In brief, the isolated

756 microsome vesicles were incubated at $25{ }^{\circ} \mathrm{C}$ for 30 minutes with trypsin (Sigma).

757 The digestion was performed in the absence or presence of $0.1 \%(\mathrm{v} / \mathrm{v})$ Triton X-

758100 and stopped by adding anti-trypsin inhibitor for 10 minutes on ice.

759 Chemical cross-linking experiments.

760 Protein cross-linking experiments were performed according to the user instruction

761 (Thermo Fisher Scientific). Briefly, for in vitro crosslink, the purified N- and C-

762 CLCC1 were incubated with DSS (Thermo Fisher Scientific) for 30 minutes at

$76325{ }^{\circ} \mathrm{C}$ then followed by adding quenching buffer ( $\left.1 \mathrm{M} \mathrm{Tris-} \mathrm{HCl}, \mathrm{pH} 8.0\right)$. We set

764 the DSS concentration gradients ranged from 0 to $1 \mathrm{mM}$. For in vivo crosslink, 
781 calcium probe ${ }^{32}$. The transfected cells were washed with HBSS buffer without

782 calcium and magnesium, and then suspended with the following buffers separately:

783 the HBSS solution containing $10 \mu \mathrm{M}$ ionomycin (Beyotime) and 1mM EGTA for

784 baseline (Fbaseline); the HBSS solution with $2 \mathrm{mM}$ calcium chloride for steady- 
785 state (Fsteady); the HBSS solution with $10 \mathrm{mM}$ calcium chloride and $10 \mu \mathrm{M}$

786 ionomycin for saturating the probe (Fmax). The relative ER steady-state $\left[\mathrm{Ca}^{2+}\right]$

787 was estimated by $\Delta$ Fsteady (Fsteady-Fbaseline) divided by $\Delta$ Fmax (Fmax-

788 Fbaseline). The fluorescent signals from individual cell were collected by

789 LSRFortessa flow cytometer (BD Biosciences). $\mathrm{For} \mathrm{Cl}^{-}$sensor and ER-GCaMP6-

790 210, we employed the FITC channel (488 nm); for DsRed, we employed PE

791 channel $(561 \mathrm{~nm})$. Data were analyzed by FlowJo X. The cells were treated

792 with 7-AAD (BioLegend) or DAPI (Beyotime) to exclude the dead cells.

\section{Transmission electron microscopy (TEM)}

794 Mice at $\mathrm{P} 30$ were perfused by $0.1 \mathrm{M}$ phosphate buffer (PB, pH 7.4) at room 795 temperature, then fixed by fixation solution (FS, 4\% PFA (W/V) in PB) and by $2.5 \%$ 796 glutaraldehyde in $\mathrm{FS}$ at $4{ }^{\circ} \mathrm{C}$ overnight. The similar regions of the cerebellums 797 were cut into $200 \mu \mathrm{m}$ for embedding which was performed at the Center for 798 Biomedical Analysis of Tsinghua University. The images were taken by Tecnai 799 Spirit electron microscopy.

800 Lentiviral shRNA knockdown and the inducible expression system.

801 Lentiviruses were produced by co-transfecting 293FT cells with transfer constructs, 802 pMD2.G and psPAX2, by linear PEI (MW 25,000, Polysciences). The medium 803 containing lentivirus without debris was concentrated by centrifugation at 20,000 804 rpm for 2 hours and resuspended in PBS. For generation of the stable cell line 


\section{Cell culture and Calcium imaging.}

815 293FT and Hela cells were maintained in Dulbecco's modified Eagle's medium 816 (DMEM) supplemented with 10\% heat-inactivated Fetal Bovine Serum (FBS) and 817 1\% penicillin/streptomycin (GE Healthcare). The primary cardiomyocyte culture 818 was performed as previously reported (PMID: 24056408). Briefly, the hearts from 819 P2 neonatal mice were dissected and minced in the $\mathrm{Ca}^{2+}$ and $\mathrm{Mg}^{2+}$ free $\mathrm{PBS}$ 820 supplemented with $20 \mathrm{mM} \mathrm{BDM}$ (Sigma). The chopped tissues were digested in 821 PBS containing $0.125 \%(\mathrm{w} / \mathrm{v})$ trypsin at $4{ }^{\circ} \mathrm{C}$ for 2 hours followed by the digestion 822 of $0.5 \%$ collagenase I (Sigma) at $37^{\circ} \mathrm{C}$ for 30 minutes. After the digestion, the 823 cardiomyocytes were seeded on gelatin (Sigma)-coated cover slips and in 824 DMEM/F12 medium containing 10\% FBS. After 48 hours, the cardiomyocytes 
825 showed spontaneous beating, which were used in calcium imaging experiments.

826 For calcium imaging, the 293FT cells or cardiomyocytes seeded on the coverslips

827 were loaded with the ratiometric $\mathrm{Ca}^{2+}$ indicator (Fura-2 AM, Thermo Fisher

828 Scientific) in Krebs-Ringer-Hepes (KRH) buffer (25 mM HEPES pH7.4, 125 mM

$829 \mathrm{NaCl}, 6 \mathrm{mM}$ glucose, $5 \mathrm{mM} \mathrm{KCl}, 1.2 \mathrm{mM} \mathrm{MgCl}$ ) supplemented with detergent

830 Pluronic F-127 (Thermo Fisher Scientific). After 30-minute loading at room

831 temperature in dark, the coverslip was washed twice with $\mathrm{KRH}$ buffer and then

832 subjected to calcium imaging in a perfusion chamber on an inverted Nikon TiE

833 microscope with $20 \times$ Fluar objective. The Metafluor Program software

834 (Molecular Devices) was used to monitor and calculate the real time changes of

835 calcium concentration in cytoplasm.

836 Western blot, immunoprecipitation, and immunostaining.

837 For western blot and immunoprecipitation (IP), the cultured cells or tissues were 838 lysed in the TBS lysis buffer (50 mM Tris-HCl pH 7.4, $150 \mathrm{mM} \mathrm{NaCl}, 1 \% \mathrm{DDM}$, and 839 protease inhibitor cocktail). After incubation for 20 minutes on ice, the cell debris 840 was removed by centrifugation at $13,000 \times \mathrm{g}$ for 5 minutes. For western blot, the 841 supernatant was boiled with $2 \times$ SDS loading buffer and the proteins were 842 separated on SDS-PAGE gel and transferred to PVDF membrane (GE Healthcare) 843 using standard protocol. The blot was incubated with the primary antibody 844 overnight at $4{ }^{\circ} \mathrm{C}$, and then HRP-conjugated secondary antibody $\mathrm{RT}$ for 60 minutes. 
845 For IP assay, the Dynabeads (Invitrogen) were used to capture the tagged target 846 proteins. The beads were washed with the TBS lysis buffer and pre-incubated 847 with the primary antibody at room temperature for 20 minutes then incubated with 848 the supernatant of the cell lysate at $4{ }^{\circ} \mathrm{C}$ for at least 3 hours or overnight. The 849 beads were washed five times with washing buffer (50 mM Tris-HCl pH 7.4, 150 $850 \mathrm{mM} \mathrm{NaCl}, 0.025 \%$ DDM, and protease inhibitor cocktail). The IPed proteins were 851 eluted by $2 \times$ SDS-loading buffer at $95{ }^{\circ} \mathrm{C}$ for 5 minutes. For cultured cell 852 immunostaining, the cultured Hela cells were fixed with $4 \%$ (W/V) 853 paraformaldehyde (PFA) and permeabilized by $0.3 \%$ Triton X-100 in PBS for 10 854 minutes. The fixed cells were blocked with blocking buffer (PBS with 3\% BSA) and stained with primary antibody overnight at $4{ }^{\circ} \mathrm{C}$, then incubated with secondary antibody for 1 hour at room temperature. For tissue immunostaining, the PFA fixed paraffin-embedded sections were deparaffinized with standard protocol as described previously ${ }^{18}$. For antigen retrieval, the section was boiled in the 859 sodium citrate buffer ( $10 \mathrm{mM}$ sodium citrate, $\mathrm{pH}$ 6.0) and cooled to room temperature. After antigen retrieval, the sections were blocked with the blocking 861 buffer and stained with the primary and secondary antibodies. For antibodies, the 862 following primary antibodies were used, including anti-FLAG (1:5000, clone 3B9 863 mouse, Abmart), anti-Myc (1:5000, clone 19C2 mouse, Abmart), anti-tubulin (1:

864 10,000, clone B-5-1-2 mouse, Sigma), anti-calmodulin binding protein (1: 2,000, 865 rabbit, Millipore), anti-Bip (1:300, rabbit, Abcam), anti-ubiquitin (1:200, P4D1 
866 mouse, Cell Signaling Technology), anti-His (1:1000, rabbit, Cell Signaling 867 Technology), and anti-GAPDH (1:5000, 14C10 rabbit, Cell Signaling Technology). 868 For secondary antibodies, we used Alexa-conjugated secondary $(488,555)$ 869 antibodies (Life Technologies; Molecular Probes) at 1:500 and HRP-linked 870 secondary antibodies (GE Healthcare) at 1: 5,000.

871 Generation of the knock-in (KI) and Clcc1 floxed mouse and genotyping.

872 For the generation of the $\mathrm{KI}$ mouse line, we synthesized the DNA oligo which 873 carried the target mutations. The gRNA (ttggttggttccaccaacaaAGG for K298A, 874 tggattggactggaagtctcTGG for S263R, and ttggcatgggtcatccttatAGG for W267R, 875 PAM sites capitalized) was generated by in vitro transcription (Invitrogen). The 876 donor DNA oligo, gRNA, and Cas9 mRNA were injected into C57BL/6J embryos. 877 The injected embryos were transferred into the oviduct ampulla of the pseudo878 pregnant ICR (JAX, Stock No. 009122) female recipients. The right genotype 879 offsprings were backcrossed to $\mathrm{C} 57 \mathrm{BL} / 6 \mathrm{~J}$ for at least three generations to 880 establish the line. For genotyping the K298A KI mouse, the gDNA PCR (forward 881 primer: ggcacagtcaaaaccaaactgatcttg and reverse primer: 882 gagcctaaaaccaaagaccagagc) products were digested with MspA1I 883 (NEB). Primers for the S263R KI mice (forward primer: ggatttgcgttcccagctcggtt 884 and reverse primer: tccgtccctttaactttgaggcag) and for the W267R KI mice (forward 885 primer: gtgggcacagtcaaaaccaaactga and reverse primer: 
886

887

888

889

890

891

892

893

894 895 effect.

896

gagcctaaaaccaaagaccagagca). The gDNA PCR products confirmed by Sanger sequencing. The animal facility at Tsinghua university has been fully accredited by the Association for the Assessment and Accreditation of Laboratory Animal Care International (AAALAC) since 2014. All animal protocols were approved by the Institutional Animal Care and Use Committee (IACUC) at Tsinghua university based on Guide for the Care and Use of Laboratory Animals (Eighth Edition, NHR).

Clcc1 floxed mouse were generated by Cyagen (China). Two loxP sites were inserted the intron 6 and 7 by CRISPR/Cas9, respectively. The founders were backcrossed to $\mathrm{C} 57 \mathrm{BL} / 6 \mathrm{~J}$ mice for at least three generations to reduce off-target

\section{Molecular Biology.}

The following sequences of CLCC1 homologues from different vertebrate species were obtained from the NCBI GenBank: Homo Sapiens (NM_001048210.2), Mus musculus (NM_145543.2), Pan troglodytes (XM_009426847.2), Rattus norvegicus (NM_133414.1), Gallus gallus (XM_422186.5), Anolis carolinensis (XM_003223596.3), Xenopus tropicalis (XM_002932173.4), Danio rerio (XM_002667211.5). The alignment result was done by using the Clustal W program and reported from http://espript.ibcp.fr/ESPript/cgi-bin/ESPript.cgi (PMID: 24753421).

\section{Mass Spectrometry.}


906 Brain lysate of K298A/NM mice were applied for IP with CLCC1-C antibody. Gel 907 bands between $55 \mathrm{kD}$ and $100 \mathrm{kD}$ from the IP were excised for ingel digestion, 908 and the WT and K298A CLCC1 small peptides were identified by mass 909 spectrometry (MS) as previously described (PMID: 24563215). Briefly, proteins 910 were disulfide reduced with $25 \mathrm{mM}$ dithiothreitol (DTT) and alkylated with $55 \mathrm{mM}$ 911 iodoacetamide. In-gel digestion was performed using sequencing grade912 modified pepsin in $1 \%$ Fomic Acid at $4{ }^{\circ} \mathrm{C}$ for $30 \mathrm{~min}$. The peptides were extracted 913 twice with $1 \%$ trifluoroacetic acid in $50 \%$ acetonitrile aqueous solution for $30 \mathrm{~min}$. 914 For LC-MS/MS analysis, peptides were separated by Thermo-Dionex Ultimate 9153000 HPLC system. The analytical column was a homemade fused silica 916 capillary column (75 $\mu \mathrm{m}$ ID, $150 \mathrm{~mm}$ length; Upchurch, Oak Harbor, WA) packed 917 with C-18 resin (300 A, $5 \mu \mathrm{m}$; Varian, Lexington, MA). Mobile phase A consisted 918 of $0.1 \%$ formic acid, and mobile phase B consisted of $100 \%$ acetonitrile and $0.1 \%$ 919 formic acid. MS/MS spectra from each LC-MS/MS run were searched against the 920 user defined database using Proteome Discoverer (Version 1.4) searching 921 algorithm. High confidence score filter (FDR < 1\%) was used to select the "hit" 922 peptides and their corresponding MS/MS spectra were manually inspected.

923 Human subjects, whole-exome sequencing (WES), and filtering of causative 924 mutations. 
925 701 sporadic ALS patients were enrolled from the Department of Neurology of

926 Peking University Third Hospital from 2007-2020. All ALS cases were diagnosed

927 as possible, probable, or definite ALS according to the revised El Escorial criteria.

928 Clinical information, including age, sex, age of onset, site of onset, disease

929 duration, family history and neurologic examination, were recorded. 1990 control

930 samples for DNA analysis were obtained from the same hospital with no diagnosis

931 of a neurological disorder. All subjects have signed the informed consent forms

932 and this study was approved by the Ethics Committee of Peking University Third

933 Hospital. For WES, DNA was isolated from peripheral blood using DNA Isolation

934 Kit (Bioteke, AU1802). Genomic DNA $(1 \mu \mathrm{g})$ were fragmented into 200-300bp

935 length by Covaris Acoustic System. The DNA fragments were then processed by

936 end-repairing, A-tailing and adaptor ligation (Agilent SureSelect Human ALL Exon,

937 V6), a 4-cycle pre-capture PCR amplification, targeted sequences capture.

938 Captured DNA fragments were eluted and amplified by post capture PCR. The

939 final products were sequenced with 150-200bp paired-end reads on Illumina HiSeq

$940 \mathrm{X}$ platform according to the standard manual. The raw data produced on HiSeq

941 X were filtered and aligned against the human reference genome (hg19) using the

942 BWA Aligner (http://bio-bwa.sourceforge.net/,v0.7.15). The single-nucleotide

943 polymorphisms (SNPs) were called by using GATK software (Genome Analysis

944 Toolkit, v3.6). Variants were annotated using ANNOVAR

945 (annovar.openbioinformatics.org/en/latest/). All variants found by the WES were 
946 further confirmed by the Sanger sequencing. Variants were filtered for presence 947 of nonsynonymous heterozygous variants with a minor allele frequency $<1 \%$ in the 948 Exome Aggregation Consortium (ExAC) database (http://exac.broadinstitute.org/), 949 the Exome Sequencing Project (ESP) (http://evs.gs.washington.edu ), the 1000 950 Genomes Project (1000G) database (http://www.1000genomes.org/) and the 951 Genome Aggregation Database (gnomAD) (http://gnomad.broadinstitute.org/). 952 To identify the functional effect of the mutations, in silico predictive programs were 953 performed, including Polyphen-2 (http://genetics. bwh.harvard.edu/pph2), SIFT-2 954 (http://sift.jcvi.org) and Mutation Taster (http:// mutationtaster.org). The genomic 955 evolutionary rate profiling scores were acquired by GERP++ program 956 (http://mendel.stanford.edu/SidowLab/downloads/gerp/index. html).

957 Quality control (QC)

958 After the variants were called and annotated, we applied QC steps to individuals 959 and variants. Briefly, individual-level QC was based on common single 960 nucleotide polymorphisms (SNPs) (minor allele frequency MAF> 1\%) with a 961 genotype call rate $>95 \%$. We excluded individuals from the association analysis 962 who (1) were sex-discordant/ambiguous (43 individuals, 17 ALS cases and 26 963 controls); (2) presented a genotyping call rate < 80\% (0 individuals); (3) exhibited 964 an excessive heterozygosity rate (> 3 SD from the mean; 36 individuals, 2 cases 965 and 34 controls); (4) were shown to be ancestry outliers based on the three 
966 principal components (PCs) derived from common SNPs (0 individuals); or (5)

967 exhibited a genetic relationship matrix value $>0.1$ with another individual (32 968 individuals, 12 ALS patients and 20 controls). After the QC procedures, a total of 969670 ALS cases and 1910 controls remained for the analyses. We performed the 970 same QC steps on the common capture set. After obtaining clean sets of 971 individuals, we excluded genetic variants based on the following criteria: (1) a low 972 genotype call rate < 99\%; (2) deviation from Hardy-Weinberg equilibrium in 973 controls $\left(p<10^{-6}\right) ;(3)$ differential missingness between cases and controls $(p<$ $\left.974 \quad 10^{-6}\right)$.

975

\section{Gene-based burden analysis}

We assessed the evidence of an excess of rare damaging mutations in the ALS cases compared to the controls at the gene level using the sequence kernel association test (SKAT)-O implemented in the R SKAT package. We used SKAT-

O because it optimally combines the burden test (which is most powerful when a high proportion of variants in a gene are causal and exhibit the same direction of effect) with SKAT (which is best used when only a small proportion of variants in a gene are causal or if both risk and protective variants are present). Briefly, we analyzed RefSeq genes with damaging singleton sets ${ }^{23}$ : missense variants with a MAF lower than $0.01 \%$ (in our dataset and East Asian populations from databases including 1000G, ESP and gnomAD non-neuro subset), and with an allele count 
986 (AC) of 1 in our data. The SKAT-O results were corrected for sex and the top ten 987 PCs based on HapMap3 SNPs. We used the default settings in the R SKAT 988 package, including the imputation of missing genotypes and resampling methods 989 for computing $p$ values.

990 END. 


\section{Figures}
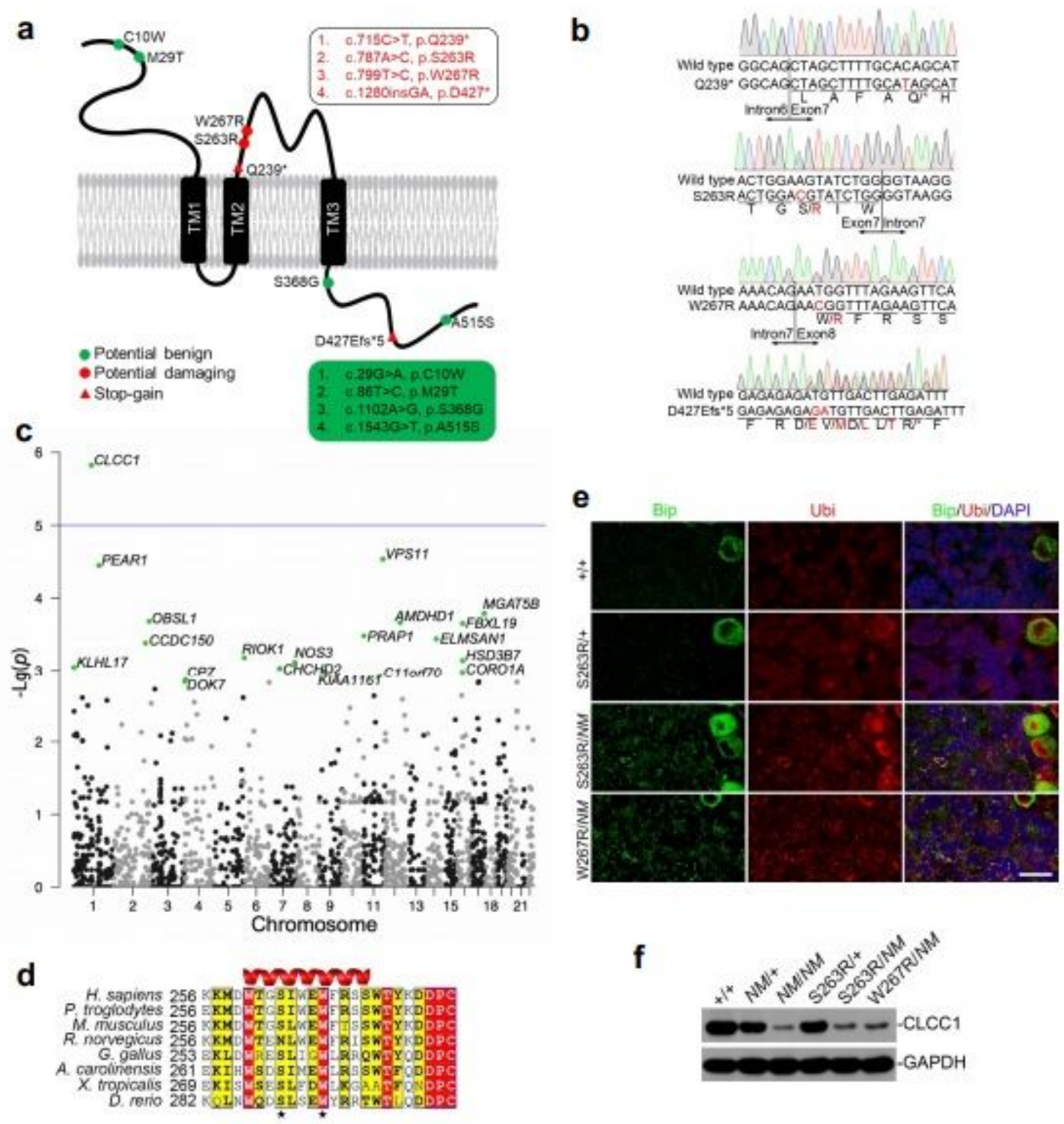

\section{Figure 1}

ALS-associated CLCC1 mutations S263R and W267R reduce mutant CLCC1 expression and promote ER stress in vivo. a, The nonsynonymous (colored circle) and stopgain (red triangle) mutations of CLCC1 were identified in a Chinese sporadic ALS cohort. The potential damaging mutations are labeled in red. $b$, Validation of the potential diseasecausing mutations of CLCC1 by Sanger sequencing. Genomic DNA extracted from peripheral blood cells of individual ALS patients. The PCR products were subject to Sanger sequencing and the boundaries of adjacent exon and intron are marked. c, The Manhattan plot for an exome-wide rare variant burden analysis. The $p$ value of $\operatorname{CLCC1}(1.51 \times 10-6$, with OR $=5.72)$. $d$, A protein alignment of CLCC1 encompassing S263, W267, and neighboring residues. Note that S263 and W267 are located in a predicted alpha helix. e, ER stress and misfolded protein accumulation documented by Bip and ubiquitin (Ubi) staining in cerebella of compound heterozygous mice (S263R/NM and W267R/NM). 
NM, the NM2453 allele is an IAP (intracisternal A-particle) insertion in the intron 2 of Clcc1, which greatly reduces the expression of CLCC1 protein to $~ 10 \%$ of that in wildtype animals (PMID: 25698737).

S263R/+ and wildtype (+/+) are negative for the phenotypes. f, Cerebellar expression of CLCC1 in the indicated genotypes. Both S263R and W267R lowered the expression of CLCC1. GAPDH, loading control. In e and $\mathrm{f},+/+, 1.5$ month; other genotypes, P35. Scale bar in $\mathrm{E}, 20 \mu \mathrm{m}$

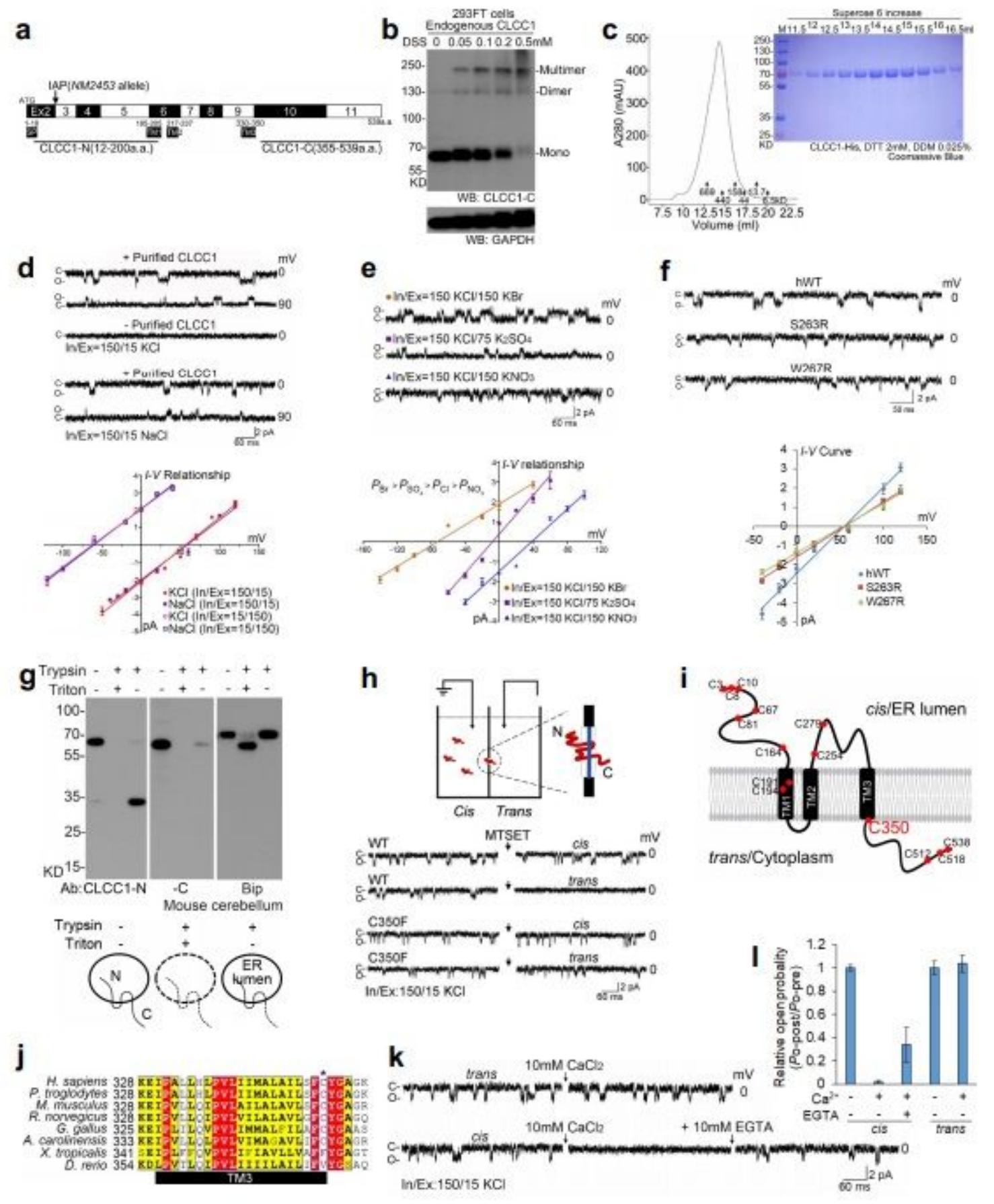

\section{Figure 2}

CLCC1 is a pore-forming component of an ER anion channel and luminal Ca2+ inhibits the channel activity. a, Domain prediction of mouse CLCC1 ( $\mathrm{mCLCC} 1$ ) that contains a signal peptide (SP) and three 
transmembrane segments (TMs). mCLCC1 is encoded by 10 exons (Ex2-11) (NM_145543.2). Note the Nterminal (12-200 a.a.) and Cterminal polypeptides (355-539 a. a.) used for generation of the $\mathrm{N}$ - and Cterminal antibodies, respectively. b, Naive 293FT cells were treated with disuccinimidyl suberate (DSS) at indicated concentrations. Cell lysates were separated by SDS-PAGE and blotted with CLCC1 C-terminal antibody (CLCC1-C). c, Chromatograph of His-tagged mCLCC1 expressed by an insect expression system and purified by Nickel column. Standard molecular weight markers are indicated by arrows. d, Purified mCLCC1 from (c) were incorporated into planar phospholipid bilayer and single channel currents were recorded in asymmetric $\mathrm{KCl}$ and $\mathrm{NaCl}$ solutions at indicated voltages (upper). C, closed state; $\mathrm{O}$, open state. Current-voltage (I-V) relationships in asymmetric $\mathrm{KCl}$ and $\mathrm{NaCl}$ solutions (lower). e, Single channel currents recorded at $0 \mathrm{mV}$ with $150 \mathrm{mM} \mathrm{KCl}$ in cis and $150 \mathrm{mM} \mathrm{KBr}, 75 \mathrm{mM} \mathrm{K} 2 \mathrm{SO}$, or $150 \mathrm{mM} \mathrm{KNO3}$ in trans (upper). I-V relationships under conditions (lower). f, Single channel activities (upper) and I-V relationships (lower) recorded from planar bilayers with purified human wildtype (hWT), S263R, and W267R mutant CLCC1, respectively. Solution, asymmetric $\mathrm{KCl}$ (In/Ex, 150/15 mM). In d, e, and f, values are presented as mean \pm SEM $(n \geq 6)$. g, Topology of CLCC1 determined by microsome preparation. Microsomal vehicles prepared from mouse cerebellum were treated with trypsin alone, or trypsin together with Triton X-100. Protein lysates were then separated by SDS-PAGE and probed with CLCC1 N- and Cterminal antibodies. As a control, Bip, an ER lumen resident, was protected from trypsinization. $\mathrm{h}$, Application of 2 mM MTSET in trans but not cis side blocked mCLCC1 channel activity. We defined cis side as the chamber we applied purified CLCC1 proteins (upper). The C350F mCLCC1 mutant was resistant to MTSET ( $n \geq 6$ ). i, Cysteine residues of CLCC1, with C350 highlighted. j, An alignment of predicted TM3 of CLCC1 across different species. C350 is labeled with an asterisk. Note the corresponding residue of Homo sapiens and Mus musculus C350 is phenylalanine in Xenopus. $k$, Application of $10 \mathrm{mM} \mathrm{CaCl} 2$ in cis but not trans reduced mCLCC1 channel activity. The inhibitory effect of $\mathrm{Ca} 2+$ was partially prevented by EDTA $(10 \mathrm{mM})$. I, Statistical analysis of normalized relative open probability (Po). Relative Po, Po-post/Po-pre, Po after $\mathrm{CaCl} 2$ or EDTA treatment divided by that before the treatment. Values are presented as mean \pm SEM $(n=6)$. 
a

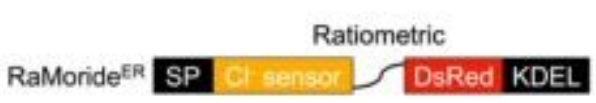

WT or mutant CLCC1 IRES miRFP670S b

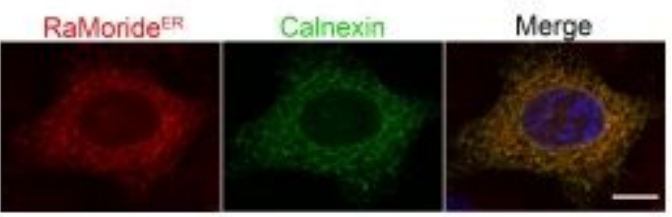

d

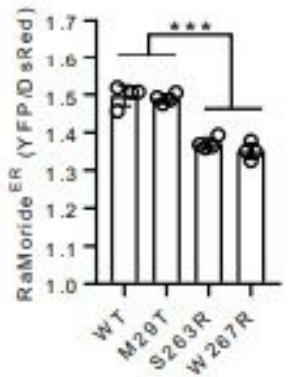

f

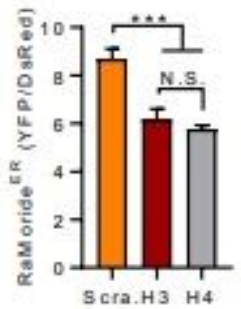

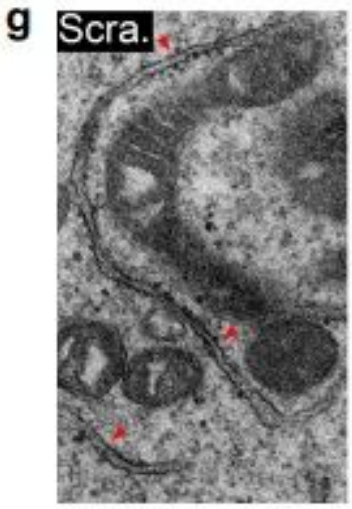
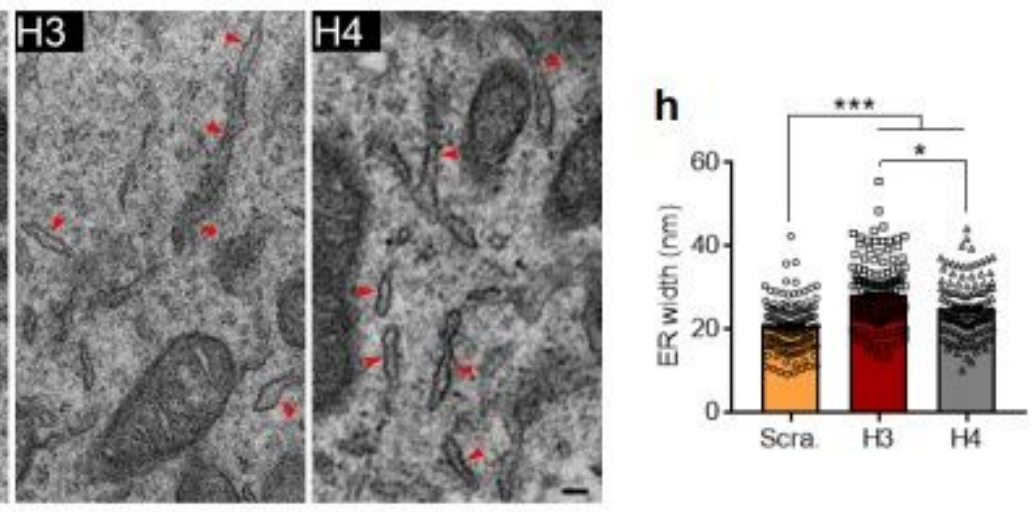

\section{Figure 3}

Dysfunction of CLCC1 impairs steady state [Cl-]ER and leads to ER swelling. a, Ratiometric Cl- sensor (RaMorideER) for ER [Cl- ] ([Cl- ]ER) measurement. A signal peptide (SP) was tagged to the $\mathrm{N}$-terminus of a previously reported Cl-sensor (PMID: 24901231), which was then fused to a monomeric DsRed as an internal control for the probe expression level and an ER retention signal (KDEL) at the C-terminal end. The resulting ratiometric Clsensor (RaMorideER) for ER [Cl- ] ([Cl- ]ER) measurement (upper). Cell expressing wildtype (WT) or ALS-associated mutant CLCC1 was monitored by the expression of an engineered near-infrared fluorescent protein, miRFP670S (PMID: 23770755). IRES (Internal Ribosome Entry Sites) sequences were employed to ensure the co-expression (lower). b, ER localization of RaMorideER, showing a prominent overlap of the DsRed fluorescence with Calnexin immunostaining signals. $c$ and d, Measurement of [Cl- ]ER in 293FT cells expressing WT or ALS-associated mutant CLCC1 
by RaMorideER. [Cl-]ER was reflected by the ratio of YFP/DsRed fluorescent signals. Cells expressing WT or ALS-associated mutant CLCC1 were sorted by miRFP670S. Representative FACS (Fluorescence Activated Cell Sorting) plots (c) and the summary data (d) are shown. e, Knockdown of CLCC1 in 293FT cells infected with lentiviral H3 and H4 shRNAs, measured by western blot (left), FACS (middle), and RNAseq (right). Ctrl., MOCK control; Scra., scrambled shRNA; H3 and H4, shRNAs specific for CLCC1. f, Steady state [Cl-]ER measured by RaMorideER in 293FT cells infected with the indicated shRNAs. $\mathrm{g}$ and $\mathrm{h}$, Transmission electron microscopy (TEM) images of 293FT cells infected with the indicated shRNAs. Ribosome-bound rough ER was marked by red arrows. ER width was calculated and the summary data are shown in (h). In $d, f$, and $h$, values are presented as mean \pm SEM from at least three independent experiments or biological replicates; N.S., no significant difference, ${ }^{*} p<0.05,{ }^{* *} p<0.01,{ }^{* \star *} p$
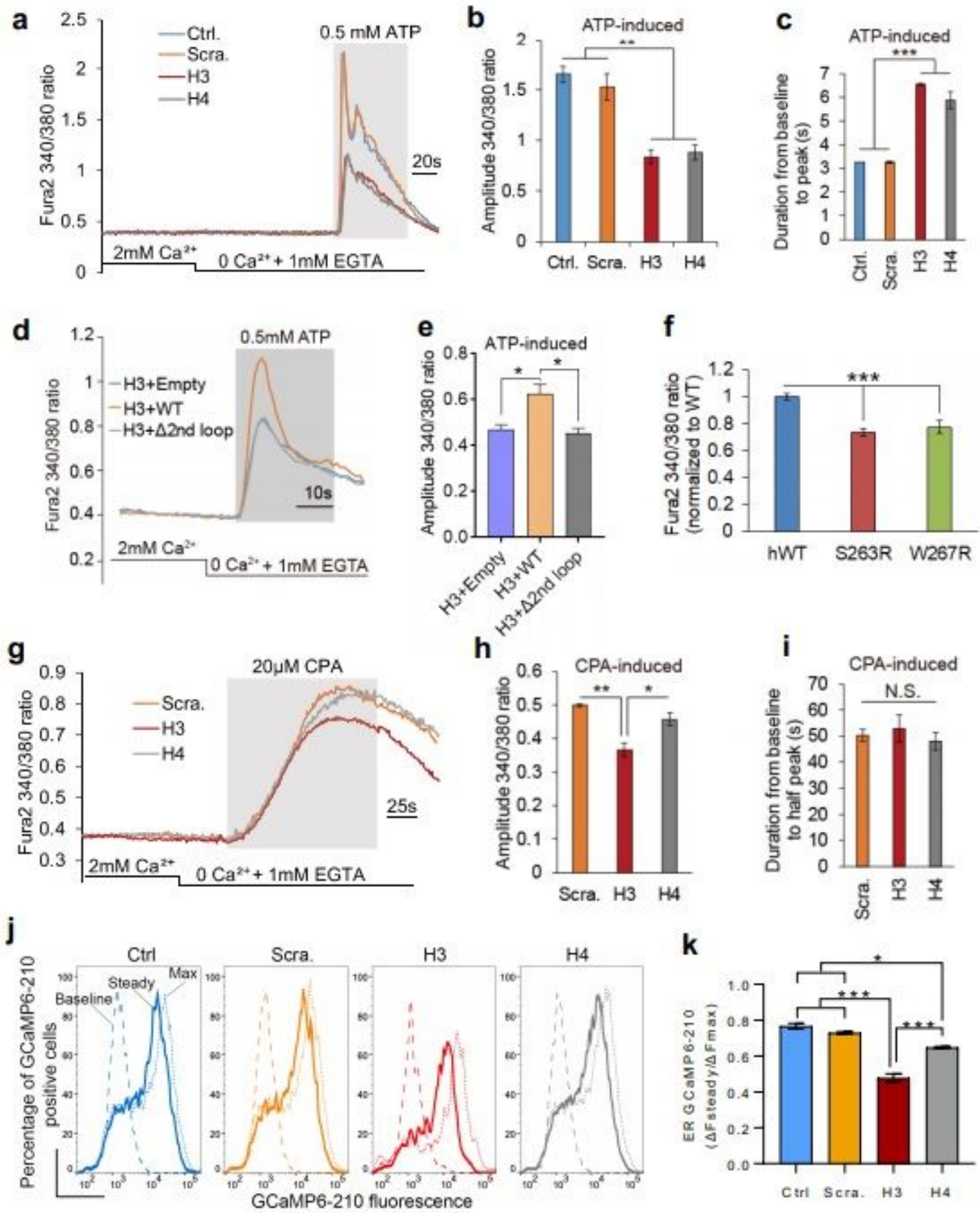

Figure 4 
Dysfunction of CLCC1 impairs internal Ca2+ release and depletion of CLCC1 dosage-dependently reduces [Ca2+]ER. a-c, 293FT cells infected with the indicated shRNAs were loaded with Fura-2 and stimulated with ATP in a calcium-free medium (gray rectangle). Representative $\mathrm{Ca} 2+$ release traces averaged from at least 50 cells (a). The knockdown of CLCC1 reduced the amplitude (b) but increased the time-to-peak (c) of ATPinduced Ca2+ release. $\mathrm{d}$ and e, Full-length WT mCLCC1 but not the $\triangle 2$ nd loop mutant CLCC1 restored the ATP-induced $\mathrm{Ca} 2+$ release damaged by $\mathrm{H} 3$ shRNA knockdown. The data summary shown in (e). f, ATP-induced internal Ca2+ release was impaired by S263R and W267R mutants. Human wildtype (hWT) and S263 and W267 mutant CLCC1 were expressed in 293FT cells. g-i, ER Ca2+ content was estimated by CPA-induced cytosolic Ca2+ rise in the calcium-free medium (gray rectangle) in 293FT cells infected with the indicated shRNAs. Shown are representative traces of CPA-induced calcium leak averaged from at least 50 cells ( $\mathrm{g}$ ) and summary data for the amplitude (h) and time-to-half peak (i) of CPA-induced cytosolic Ca2+ rise. j and k, Steady state [Ca2+]ER in 293FT cells infected with the indicated shRNAs was measured by fluorescent signals of ER-GCaMP6-210 a previously reported low affinity Ca2+ probe (PMID: 28162809) by FACS. Baseline, 1 mM EGTA + $10 \mu \mathrm{M}$ ionomycin; Steady, normal medium containing $2 \mathrm{mM} \mathrm{Ca} 2+$; Max, $10 \mathrm{mM} \mathrm{Ca} 2++10 \mu \mathrm{M}$ ionomycin. The summary data $(\mathrm{k})$ were from three independent experiments. $\Delta$ Fsteady $=($ Fsteady-Fbaseline $) ; \Delta \mathrm{Fmax}=($ Fmax-Fbaseline $)$. Values are presented as mean \pm SEM. In $b, c, e, f, h$, and $i, n>150$ cells pooled from three independent experiments. N.S., no significant difference, ${ }^{\star} p<0.05,{ }^{\star *} p<0.01,{ }^{* \star *} p$ 

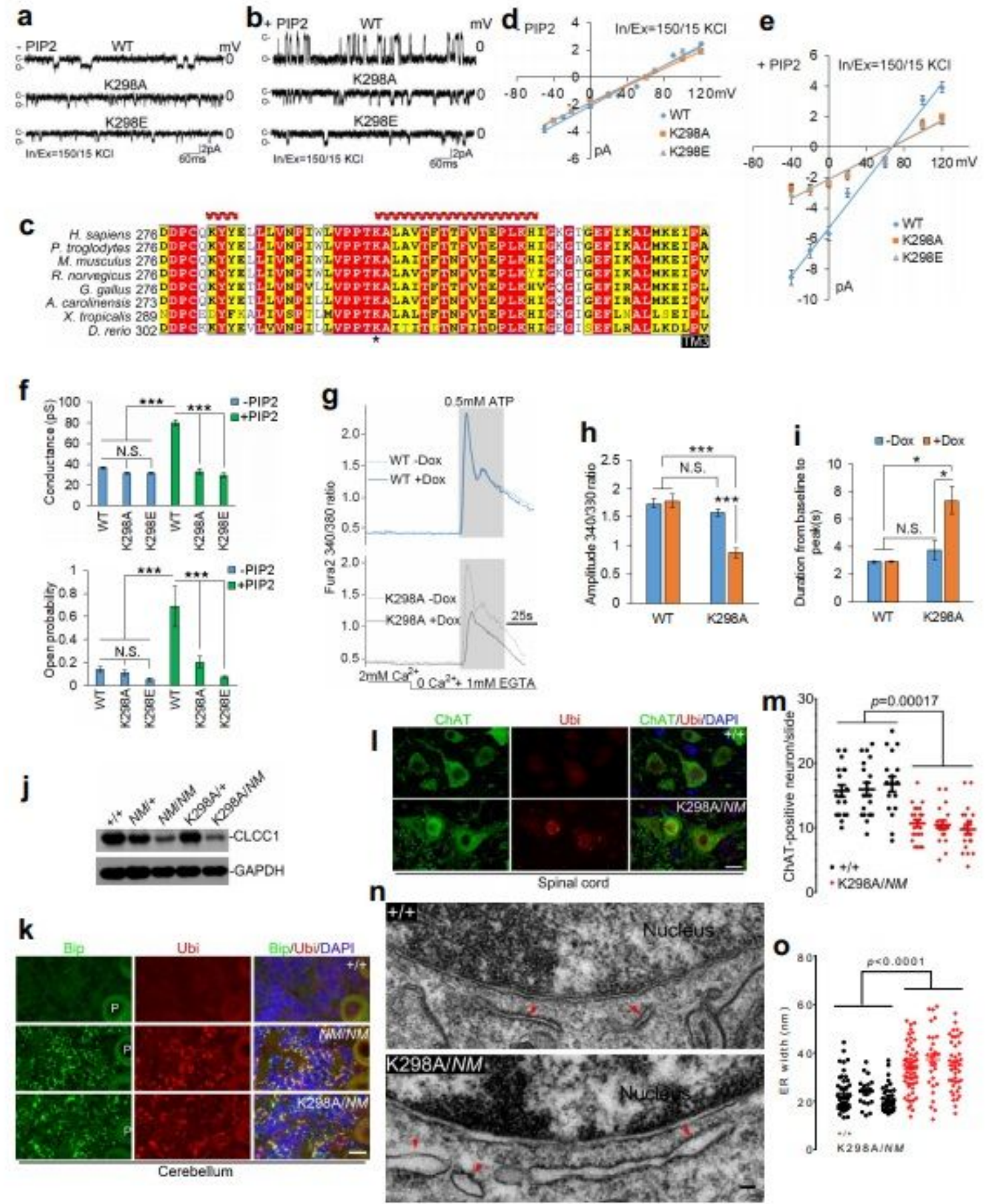

\section{Figure 5}

Mutation of K298 a PIP2-sensing residue for CLCC1 channel activity impairs ER Ca2+ release and promotes ER swelling and motor neuron degeneration. $a$ and $b$, Single channel activities recorded after incorporating the purified wildtype (WT), K298A, and K298E mutant mCLCC1 into the planar phospholipid bilayer. In b, the phospholipid bilayer contained 2\% PIP2. c, An alignment of sequences encompassing the 2nd loop of CLCC1 among different species. $d$ and e, I-V relationships in the absence (d) and presence (e) of PIP2 for WT mCLCC1 and its K298A and K298E mutants recorded from planar phospholipid bilayer in 
the asymmetric $\mathrm{KCl}$ solutions. $\mathrm{f}$, Summary data of slope conductance (upper) and channel open probability ( $\mathrm{Po}$ ) at $0 \mathrm{mV}$ (lower) in the asymmetric $\mathrm{KCl}$ solutions. g, A lentiviral inducible system was used to express wildtype mCLCC1 (WT) and its K298A mutant (K298A) in 293FT cells. ATP-induced Ca2+ release was measured in the calcium-free culture medium in Fura-2 loaded cells with (+Dox) or without (Dox) induction. $\mathrm{h}$ and i, Summary data of amplitude (h) and time-to-peak (i) of ATP-induced Ca2+ release under conditions shown in (g). j, Cerebellar expression of CLCC1 in the indicated genotypes. GAPDH, loading control. k, ER stress and misfolded protein accumulation documented by Bip and ubiquitin (Ubi) staining, respectively, in cerebella of NM2453 homozygotes (NM/NM) and K298A and NM2453 compound heterozygotes (K298A/NM). P, Purkinje cells. Wildtype (+/+), negative control; NM2453 homozygotes (NM/NM), positive control. I and m, Ubiquitinpositive inclusions in ChAT-positive motor neurons in lumbar 4-5 spinal cords of K298A/NM mice. Representative images ( $\mathrm{l}$ ) and quantification of number of ChAT-positive motor neurons in the ventral horn $(\mathrm{m})$ are shown. $\mathrm{n}$ and $\mathrm{o}$, TEM images of cerebellar granule neurons from wildtype $(+/+)$ and K298A/NM mice (n). Red arrows indicate ribosomebound rough ER. Summary data are shown in (o). Mouse age: $j$ and k, 1.5 month; I and m, $+/+, 10$ months, K298A/NM, 14 months; $n$ and o, 3 months. Quantification: d, e, and f, $n=4-20 ; h$ and i, more than 150 cells from three independent experiments; $m$ and o, 14-18 slides $(\mathrm{m})$ and more than 25 granule cells (o) per mouse from three individual animals for each genotype. Scale bar, $k, 20 \mu \mathrm{m} ; \mathrm{I}, 10 \mu \mathrm{m} ; \mathrm{n}, 50 \mathrm{~nm}$. Values are presented as mean $\pm S E M ;$ N.S., no significant difference; ${ }^{*} p<0.05 ;{ }^{* \star *} p<0.001$, by $t$-test or oneway ANOVA. 


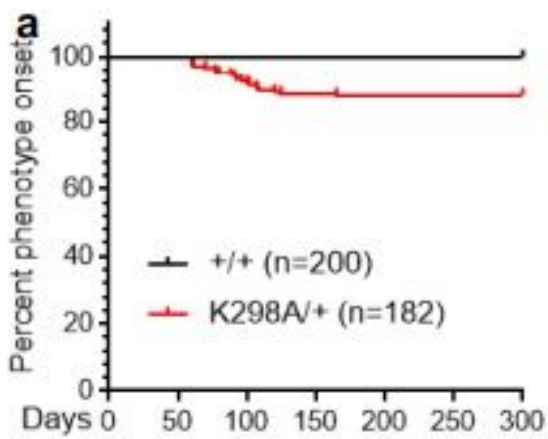

b

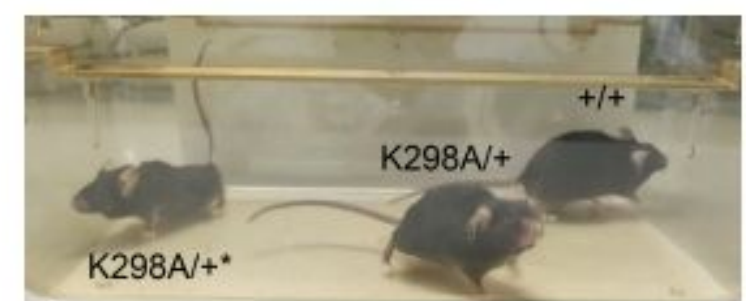

C

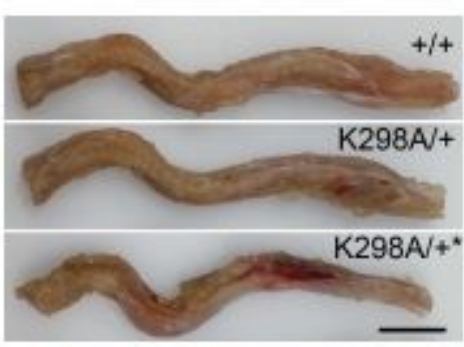

d
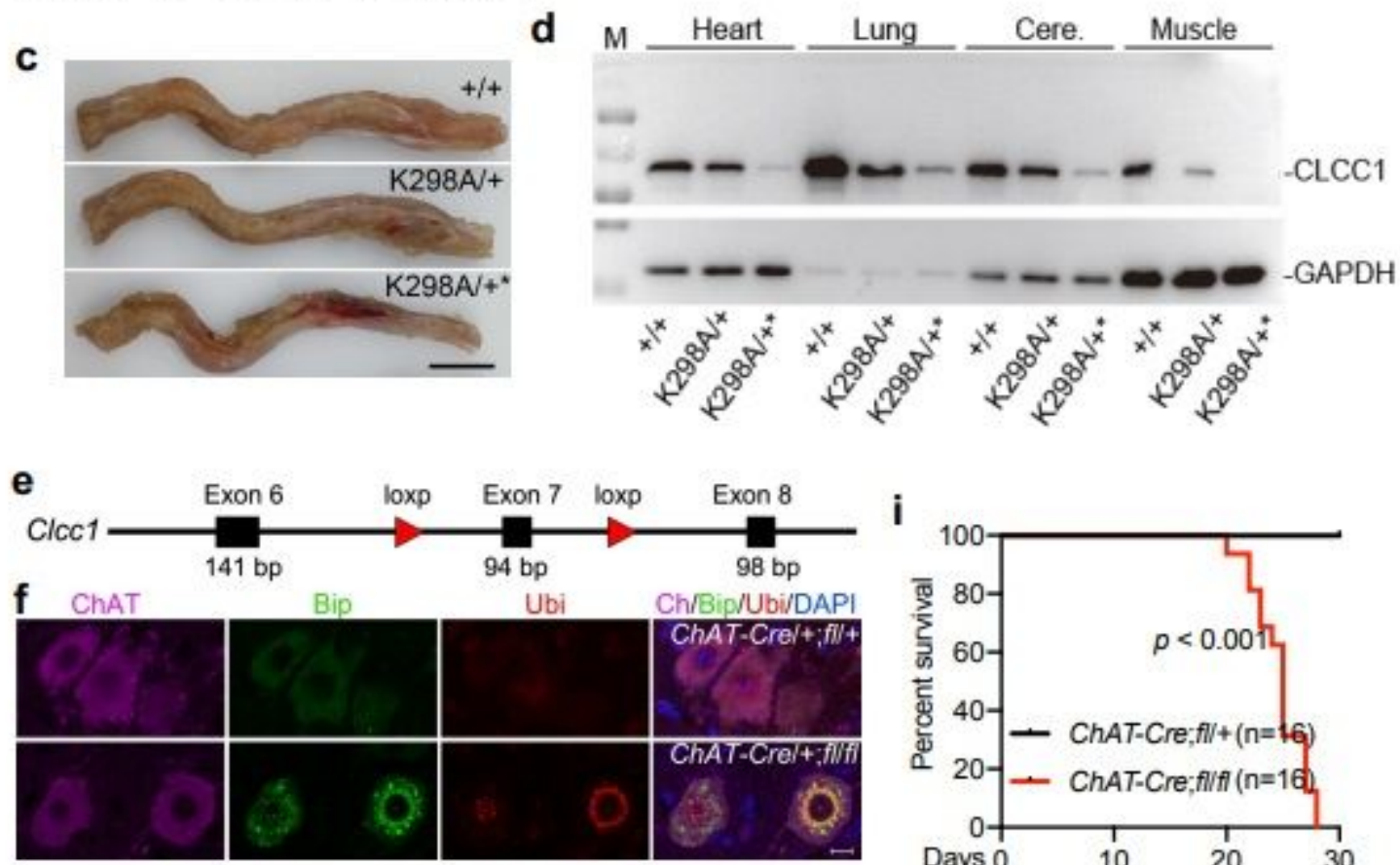

Exon 6

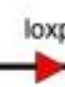

Exon 7 loxp Exon 8

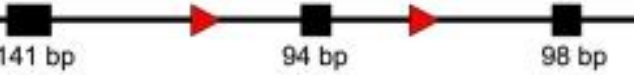

Ubi
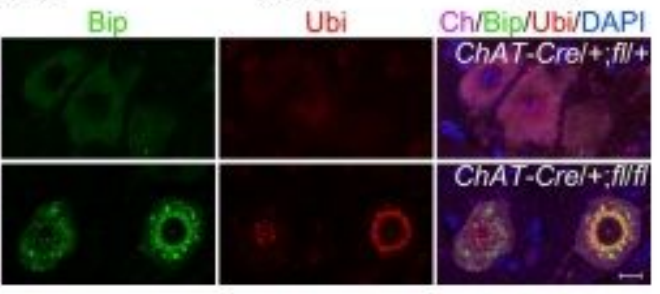

g ChAT

Ubi

ChAT/ERp72JUbi

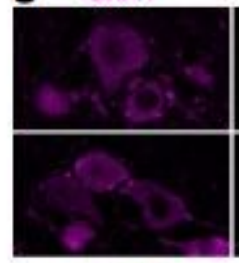

ERp72
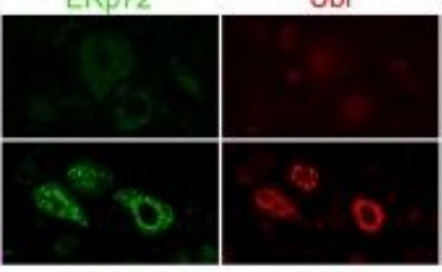

ChAT-Cre/+;ill +

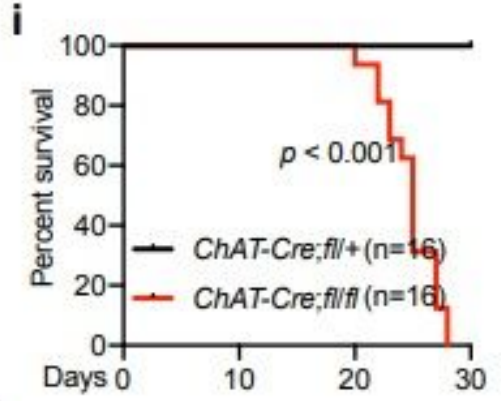

h ChAT

TDP-43
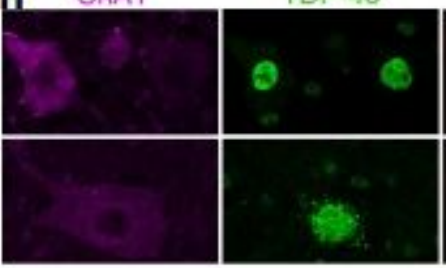

Ubi

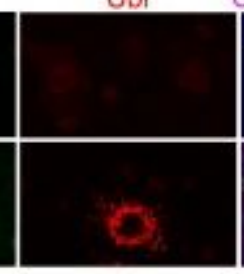

Ch/TDPJUi/DAP

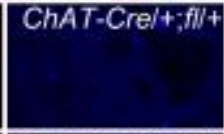

ChAT-Crelt; fllifi

sing

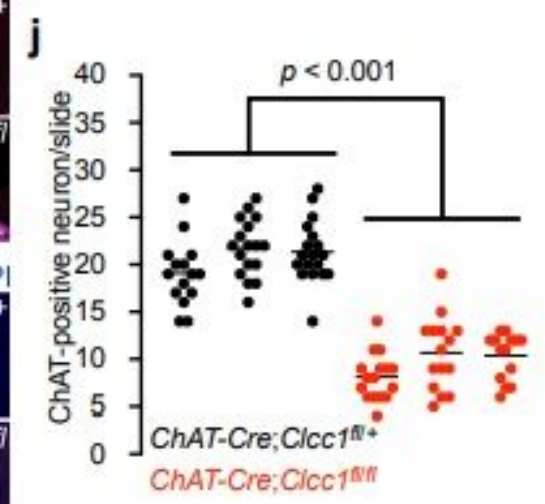

Figure 6

Increased penetrance of K298A allele and cell-autonomous effect of Clcc1 loss-of-function in motor neuron loss. a, Percent of phenotype onset in wildtype (+/+) and K298A/+ animals. The early onset phenotypes of $\mathrm{K} 298 \mathrm{~A} /+$ animals $\left(\mathrm{K} 298 \mathrm{~A} /+^{*}, 12 / 182\right.$, onset time: postnatal $90.9 \pm 5.5$ days) include body weight loss, hindlimb weakness, trunk shaking, tail flagging, abnormal gaits, and ataxia (also see Movie S2). b, A K298A/+ mutant mouse displaying early onset phenotype (K298A/+*) together with a wildtype 
and a normal K298A/+ mouse. c, Curved spine shown in K298A/+* but not in wildtype and K298A/+ mice. d, Expression levels of CLCC1 in various tissues in wildtype, $K 298 \mathrm{~A} /+$, and $\mathrm{K} 298 \mathrm{~A} /+^{\star}$ mice. Summary data in Extended Data Fig. 13. e, Construction of Clcc1 conditional knockout mouse. $\mathrm{f}-\mathrm{j}$, Knockout of Clcc1 in ChAT-positive motor neurons (ChAT-Cre;Clcc1fl/fl) leads to ER stress ( $\mathrm{fand} g$ ), TDP43 pathology (h), early death (i), and motor neuron loss (j). ChAT-Cre;Clcc1 fl/+ served as a negative control. In j, 14-18 slides per mouse from lumbar 4-5 spinal cords of three individual animals for each genotype were analyzed. Mouse age: b-d, 10 months; f, g, h, and j, P20-25. Scale bar in f-h, $10 \mu \mathrm{m}$.

\section{Supplementary Files}

This is a list of supplementary files associated with this preprint. Click to download.

- SupplementaryMovie1LinkCLCC1toALS.mp4

- SupplementaryMovie2LinkCLCC1toALS.mp4

- FiguresLinkCLCC1toALS0516.pdf 